\title{
Compensating tune spread induced by space charge in bunched beams
}

\author{
Vladimir N. Litvinenko, ${ }^{1,2, *}$ and Gang Wang ${ }^{2}$ \\ ${ }^{1}$ Department of Physics and Astronomy, Stony Brook University, \\ Stony Brook, New York 11794, USA \\ ${ }^{2}$ Collider Accelerator Department, Brookhaven National Laboratory, \\ Upton, New York 11973, USA \\ (Received 14 May 2014; published 20 November 2014)
}

\begin{abstract}
The effects of space charge play a significant role in modern-day accelerators, frequently constraining the beam parameters attainable in an accelerator or in an accelerator chain. They also can limit the luminosity of hadron colliders operating either at low energies or with sub-TeV high-brightness hadron beams. The latter is applied for strongly cooled proton and ion beams in eRHIC - the proposed future electron-ion collider at Brookhaven National Laboratory. Several schemes were proposed to compensate for space-charge effects in a coasting (e.g., continuous) hadron beam, and some have been tested. Using an appropriate transverse profile of the electron beam (or plasma column) for a coasting beam would compensate both the tune shift and the tune spread in the hadron beam. But none of these methods address the issue of compensating space-charge induced tune spread in a bunched hadron beam, i.e., the dependence of the particle's tune shift on its longitudinal position inside the bunch. In this paper we propose and evaluate a novel idea of using a copropagating electron bunch with mismatched longitudinal velocity to compensate the space-charge induced tune shift and tune spread. We present several practical examples of such a system.
\end{abstract}

DOI: 10.1103/PhysRevSTAB.17.114401

PACS numbers: 29.20.db, 29.27.-a

\section{INTRODUCTION}

The paper is motivated by developing a high-energy high-luminosity electron-ion collider at Brookhaven National Laboratory called eRHIC [1], where $21.2 \mathrm{GeV}$ polarized electrons will collide with proton and ion beams, having energies from 50 to $250 \mathrm{GeV} / \mathrm{u}$. The eRHIC plans to use a very short (with rms bunch length of $5 \mathrm{~cm}$ ) and strongly cooled proton and ion beams with normalized transverse emittances of $0.2 \mathrm{~mm}$ mrad. Cooling these hadron beams with high peak current to such low emittances would generate a significant betatron tune shift and spread induced by the space charge. Hence, the goal of this paper is to propose a technique, which could be used to mitigate negative space-charge effects using a compensation scheme applicable to bunch beams.

Space-charge effects have been known in accelerator physics for half of the century. There is extensive literature [2-22] (with an excellent and concise review by Zotter [23]) describing the effect of space charge on the beam's quality and stability. A nonlinear space-charge force induces an irreducible transverse tune spread, i.e., the tune

\footnotetext{
* Corresponding author. vladimir.litvinenko@stonybrook.edu

Published by the American Physical Society under the terms of the Creative Commons Attribution 3.0 License. Further distribution of this work must maintain attribution to the author(s) and the published article's title, journal citation, and DOI.
}

dependence on both the hadron's ${ }^{1}$ longitudinal position inside the bunch, $z$, and the amplitude of the transverse oscillations.

It is well known that space-charge effects fall as a high power of the beam's relativistic factor:

$$
\Delta Q_{\mathrm{sc}} \approx-\frac{Z^{2} r_{p}}{A} \frac{N_{o}}{4 \pi \beta_{h}^{2} \gamma^{3} \varepsilon} \frac{C}{\sqrt{2 \pi} \sigma_{z}}
$$

where $C$ is the ring's circumference, $Z$ is the charge, and $A$ is the atomic number of the hadron (e.g., an ion, for proton $Z=A=1), r_{p}=e^{2} / m_{p} c^{2}$ is the classical radius of the proton, $\gamma^{2}=1 /\left(1-\beta^{2}\right)$ is the relativistic factor of hadron beam, $N_{o}$ is number of hadrons in the bunch with an rms bunch length of $\sigma_{z}$, and $\varepsilon$ is the beam's transverse emittance.

While space-charge effects exist in any charged beam, they have stronger implications for hadron beams. Hadrons become ultrarelativistic at much higher energies, and also travel a longer pass in accelerators, compared to their lepton counterpart, to become ultrarelativistic.

\footnotetext{
${ }^{1}$ Here we are considering only positively charged particles, the space-charge effects in which can be compensated by using negatively charged electron beam. Compensation for negatively charged particles, including antiprotons and negatively charged ions, would require positively charged particles. Using positron, proton, or ion beams for compensating the space charge in the negatively charged beams, while theoretically possible, most likely is impractical.
} 
One of the most important effects is the tune spread induced by the intrinsically nonlinear space-charge force. ${ }^{2}$ Appendix A gives the general expression of the tune spread. Since the compensation scheme we are presenting here does not depend on the details of the transverse beam's distribution, e.g., similarly to the compensation techniques suggested for coasting beams, the transverse profile of the electron beam (or a column) should compensate both the tune shift and its dependence on the amplitudes of the transverse oscillations [24-28]. Naturally, the maximum tune shift is experienced by the particles in the center of the beam, while the particles with large amplitude of oscillations experience a smaller value of the tune shift. The overall tune spread is determined by its value for the center particles.

Thus, for simplicity we consider here a hadron beam with equal transverse emittances, $\varepsilon_{x}=\varepsilon_{y}=\varepsilon$, whose tune shifts are given by (A26-A28):

$$
\begin{aligned}
\delta Q_{x, y} & =\delta Q_{\mathrm{sc}}(z) \cdot f_{x, y} ; \\
\delta Q_{\mathrm{sc}}(z) & =-\frac{C}{4 \pi \varepsilon} \frac{1}{\beta^{2} \gamma^{3}} \frac{Z^{2} r_{p}}{A} \cdot \frac{N_{o}}{\sqrt{2 \pi} \sigma_{z}} \cdot e^{-\frac{z^{2}}{2 \sigma_{z}^{2}}} ; \\
f_{x} & =\left\langle\frac{2}{1+\sqrt{\beta_{y} / \beta_{x}}}\right\rangle ; \quad f_{y}=\left\langle\frac{2}{1+\sqrt{\beta_{x} / \beta_{y}}}\right\rangle .
\end{aligned}
$$

Since the longitudinal motion of hadrons usually is very slow (e.g., $Q_{s} \ll Q_{x, y}$ ), the tune of the particle depends not only on the amplitudes (actions) of the transverse oscillations, but also on their longitudinal location within the bunch.

One practically important feature of the space-charge effects is a very strong dependence on the relativistic factor, $\gamma: \delta Q_{\mathrm{sc}} \propto \gamma^{-3} /\left(1-\gamma^{-2}\right)$. While the power one of $\gamma$ naturally comes from the increasing rigidity of the beam, the $\gamma^{-2}$ comes from the effective canceling of the forces from the electric and magnetic fields induced by the beam $(\text { details are given in Appendix } \mathrm{A})^{3}$ :

$\vec{F}_{\perp}=e Z\left(\vec{E}_{\perp}+\beta_{o}\left[\hat{z} \times \vec{B}_{\perp}\right]\right)=e Z \cdot \vec{E}_{\perp}\left(1-\beta_{o}^{2}\right) \equiv \frac{e Z \cdot \vec{E}_{\perp}}{\gamma^{2}}$

Several practical schemes were suggested for spacecharge tune shift and tune spread compensation by

\footnotetext{
${ }^{2}$ In this paper, for compactness, we assume a Gaussian longitudinal distribution of particles. Naturally, the treatment presented herein can be extended to other types of longitudinal distributions.

${ }^{3}$ Please note that in this paper we are using Gaussian units, where, for example, both electric and magnetic fields are measured in Gs.
}

colliding an electron beam with hadron beam (e.g., an electron lens), or employing an electron column induced in a residual gas [24-28]. The tune shift given by the colliding beam does not suffer from $\gamma_{h}^{-2}$ cancellation. Vice versa, it is amplified. For an electron lens with round electron beam having rms size of $\sigma_{e}$ and the interaction length of $L$, the tune shift is given by the following:

$$
\begin{aligned}
\delta Q_{x, y e l} & =\frac{Z}{A} \frac{1}{\beta_{h} \gamma_{h}} \frac{r_{p}}{4 \pi \sigma_{e}^{2}} \cdot \frac{I_{e}}{e c \beta_{e}}\left(1+\beta_{e}{ }^{2}\right) \cdot L\left\langle\beta_{x . y}\right\rangle ; \\
L\left\langle\beta_{x . y}\right\rangle & \equiv \int_{0}^{L} \beta_{x . y} d z
\end{aligned}
$$

where $\beta_{e}=\mathrm{v}_{e} / c$ is the normalized velocity and of the electron beam. ${ }^{4}$ Comparing Eqs. (2) and (4) one can conclude that electron beam's current,

$$
\begin{aligned}
I_{e} & =\frac{C}{2 \gamma_{h}^{2} L} \cdot \frac{2 \beta_{e}}{\beta_{h}\left(1+\beta_{e}^{2}\right)} \cdot \frac{\sigma_{e}^{2}}{\left\langle\beta_{x . y}\right\rangle \varepsilon_{h}} \cdot I_{p} \propto \frac{C}{2 \gamma_{h}^{2} L} \cdot \frac{\beta_{e}}{\beta_{h}} \cdot I_{p}, \\
I_{p} & =\frac{e c Z N_{o} \beta_{h}}{\sqrt{2 \pi} \sigma_{z}} ; \quad \frac{\sigma_{e}^{2}}{\left\langle\beta_{x . y}\right\rangle \varepsilon_{h}} \sim 1,
\end{aligned}
$$

can be used to compensate for the space-charge induced tune shift. Invariably, the interaction length is much smaller than the ring's circumference e.g., $\eta=L / C \ll 1$. We can compensate for this shortcoming by having large relativistic factor, $2 \gamma_{h}^{2} \beta_{h} / \beta_{e} \gg 1$. This means that the electron current in such an electron lens can be modest, and frequently, it can be comparable to the hadron beam's current.

As explained in [24,28], by selecting a proper transverse distribution of electron beam, we can match the dependence of the space-charge tune shift on the transverse amplitudes. The only, but important, shortcoming of this method is that the tune shift introduced by the electron lens (or the column) is identical for all particles, independent of their longitudinal location inside the bunch.

However, in a bunched beam, the space-charge tune shift depends on the hadron's position within the hadron bunch, $z$. Thus, the $z$-dependence of the tune shift cannot be compensated for by using an electron lens or an electron column. Thus, for a bunched beam, at best these schemes could reduce the space-charge tune spread by a half, e.g., by compensating for it at the half of the peak value at $z=0$.

Using a copropagating electron beam with the same relativistic velocity $\beta_{e}=\beta_{h}$ (as in electron cooling schemes) and the same longitudinal distribution offers an opportunity of compensating for both the transverse and

\footnotetext{
${ }^{4}$ We would like to point to the possible confusion caused by the multiple usage of the symbol $\beta$. Unfortunately, it is unavoidable when both the velocities $\beta=\mathrm{v} / c$ and the lattice functions $\beta_{x, y}$ must be used in the same paper.
} 
longitudinal dependences of the space-charge field. Unfortunately, the compensating beam suffers from $\gamma_{h}^{-2}$ cancellation, and such a scheme would require a very large electron beam current:

$$
I_{e} \approx \frac{C}{L} \cdot I_{p} \gg I_{p} .
$$

This unfavorable scaling makes such a scheme impractical, especially for hadron beams in large colliders. For example, eRHIC would be operating hadron beams with peak current $\sim 10 \mathrm{~A}$ (and duration of $0.4 \mathrm{nsec}$ ). Using a $30 \mathrm{~m}$ of the $3.8 \mathrm{~km}$ RHIC circumference for such a spacecharge compensator would require having an electron bunch with a peak current $\sim 1.2 \mathrm{kA}$, and the bunch charge $\sim 4000$ nC. Such an e-beam simply does not exist.

We propose to use the copropagating scheme, but with mismatched relativistic factors (e.g., velocities) of the two beams. Such mode offers the possibility of diminishing the reduction factor while keeping under control the slippage between the beams.

\section{THE IDEA UNDERLYING THE METHOD}

The idea for the proposed method is based on a simple observation that the relativistic canceling is proportional to $\gamma^{2}$, while the velocity of the particles weakly depends on $\gamma$ for $\gamma>2$. To be exact, we consider a copropagating relativistic e-beam having a nearly identical bunch profile as the hadrons, but having a different relativistic factor.

Let us consider first the simple case of both beams being round and relativistic, i.e., the velocities of both beams are close to the speed of light. Hence, the slippage of the e-beam with the respect to the hadron beam is small compared with the length of the interaction section, $L$ :

$$
\Delta z=\left(\mathrm{v}_{e}-\mathrm{v}_{h}\right) \tau=\frac{L}{\beta_{h}}\left(\beta_{e}-\beta_{h}\right) \approx \frac{L}{2}\left(\frac{1}{\gamma_{h}^{2}}-\frac{1}{\gamma_{e}^{2}}\right) .
$$

For long bunches with $\gamma \sigma_{z} \gg \sigma_{\perp}$, the transverse fields induced by beams can be easily calculated using the Gauss or Ampere law:

$$
\begin{aligned}
& B_{h \theta}(r, z, s)=\frac{2 I_{o}(z)}{c r} \int_{0}^{r} f_{h}(x, s) x d x ; \\
& E_{h r}(r, z, s)=-\frac{B_{h \theta}(r, z, s)}{\beta_{h}} ; \\
& B_{e \theta}(r, z, s)=\frac{2 I_{e}(z)}{c r} \int_{0}^{r} f_{e}(x, s) x d x ; \\
& E_{e r}(r, z, s)=-\frac{B_{e \theta}(r, z, s)}{\beta_{e}},
\end{aligned}
$$

where $f_{e, h}(r, s)$ are transverse distributions of the beams. The force acting on the hadron from the e-beam is

$$
\begin{aligned}
F_{r}= & Z e\left(-\frac{B_{e \theta}(r, z, s)}{\beta_{e}}+\beta_{h} B_{e \theta}(r, z, s)\right) \\
= & -\frac{Z e}{\beta_{e}} B_{e \theta}(r, z, s) \cdot\left(1-\beta_{e} \beta_{h}\right) \\
= & -Z e \frac{2 I_{e}(z)}{c r} \cdot \frac{1-\sqrt{\left(1-\gamma_{e}^{-2}\right)\left(1-\gamma_{h}^{-2}\right)}}{\sqrt{\left(1-\gamma_{e}^{-2}\right)}} \\
& \times \int_{0}^{r} f_{e}(x, s) x d x .
\end{aligned}
$$

For ultrarelativistic particles (8) becomes

$$
F_{r} \cong-Z e \frac{I_{e}(z)}{c r} \int_{0}^{r} f_{e}(x, s) x d x \cdot\left(\frac{1}{\gamma_{e}^{2}}+\frac{1}{\gamma_{h}^{2}}\right)
$$

while self-action gives

$$
F_{r \mathrm{sc}} \cong Z e \frac{I_{h}(z)}{c r} \int_{0}^{r} f_{h}(x, s) x d x \cdot \frac{2}{\gamma_{h}^{2}} .
$$

Thus, for unequal velocities, the relativistic $\gamma_{h}^{-2}$ cancellation is replaced by $\left(\gamma_{e}^{-2}+\gamma_{h}^{-2}\right) / 2$.

Since using low-energy electron beams is economically favorable, let us assume that the relativistic factor of the hadron is significantly larger than that of the e-beam:

$$
\gamma_{h}^{2} \gg \gamma_{e}^{2}
$$

This assumption makes $\frac{1}{\gamma_{e}^{2}} \gg \frac{1}{\gamma_{h}^{2}}$ and simplifies (7) and (9):

$$
\begin{aligned}
F_{h r} & \cong-Z e \frac{2 I_{h}(z)}{\gamma_{h}^{2} c r} \int_{0}^{r} f_{h}(x, s) x d x ; \\
F_{e r} & \cong-Z e \frac{I_{e}(z)}{\gamma_{e}^{2} c r} \int_{0}^{r} f_{e}(x, s) x d x ; \\
\Delta z & \cong-\frac{L}{2 \gamma_{e}^{2}} .
\end{aligned}
$$

Let us also assume that the e-beam has the same transverse shape, as does the hadron beam. Then, as follows from Eq. (11), compensating for the space-charge effects accumulated by the hadron beam in the ring with circumference $\mathrm{C}$, we will need the electron beam current of

$$
I_{e} \cong-2 I_{h} \cdot \frac{\gamma_{e}^{2}}{\gamma_{h}^{2}} \cdot \frac{C}{L}
$$

If we further assume that it could slip for one rms bunch length of the proton bunch (practical examples are given in 
the following section) during the interaction, e.g., the length of interaction section is

$$
L \sim 2 \gamma_{e}^{2} \sigma_{z}
$$

Then, the ratio between the beam currents becomes independent of the electron's energy:

$$
\frac{I_{e}}{I_{h}} \cong \frac{C}{\sigma_{z} \gamma_{h}^{2}}
$$

and the latter should be determined by a practical matters of building an electron accelerator. Naturally, there can be $N_{c} \geq 1$ space-charge compensation sections, ${ }^{5}$ which proportionally reduce the required e-beam current:

$$
\frac{I_{e}}{I_{h}} \cong \frac{C}{N_{c} \sigma_{z} \gamma_{h}^{2}} .
$$

For example, for $\gamma_{h}=100$ and the RHIC circumference $C=3.8 \mathrm{~km}$ the required ratio is the following:

$$
\frac{I_{e}}{I_{h}} \sim \frac{1}{N_{c}} \cdot \frac{1}{\sigma_{z}(n \mathrm{sec})} .
$$

This means that electron beam's peak current can of the same order as that of the hadrons.

\section{THE METHOD}

We now consider the method for finite velocities without any limitations, and an electron beam having longitudinal profile determined by its current

$$
I_{\mathrm{e}}\left(t-\frac{s}{\mathrm{~V}_{\mathrm{e}}}\right) .
$$

As shown in Fig. 1, it merges with the hadron beam, copropagates along the interaction region from $s=0$, and is taken out at $s=L$. A hadron passes the interaction region as follows:

$$
s=\mathrm{v}_{h} \cdot\left(t-t_{o}\right) ; \quad t=t_{o}+\frac{s}{\mathrm{v}_{h}},
$$

and is affected by the electron beam's current of

$$
I_{\mathrm{e}}\left(t_{o}+\frac{s}{\mathrm{v}_{h}}-\frac{s}{\mathrm{v}_{e}}\right)
$$

The integrated effect is denoted by the following expression:

\footnotetext{
${ }^{5}$ As we discuss later, it even can be beneficial.
}

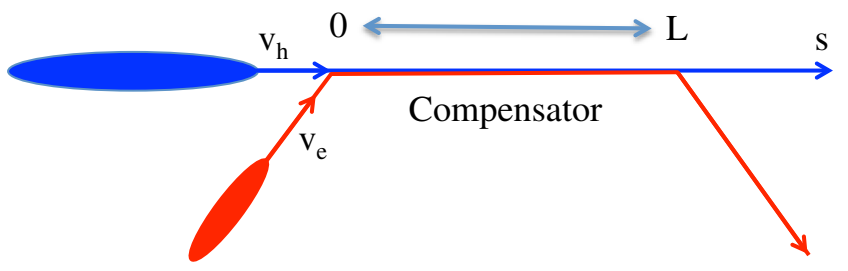

FIG. 1. A generic layout of the interaction region of the spacecharge compensator. Electron beam merges with the hadron beam, copropagates through the straight section with length $L$, and then is extracted. Their velocities $\mathrm{v}_{e} \equiv c \beta_{e}$ and $\mathrm{v}_{h} \equiv c \beta_{h}$ can differ, e.g., the beams can slide with respect to each other.

$$
\begin{aligned}
L \cdot \bar{I}_{\mathrm{e}}(t) & =\int_{o}^{L} I_{\mathrm{e}}\left(t+\frac{s}{\mathrm{v}_{h}}-\frac{s}{\mathrm{v}_{e}}\right) d s \\
& \equiv c \frac{\beta_{e} \beta_{h}}{\beta_{h}-\beta_{e}} \int_{o}^{\Delta t} I_{\mathrm{e}}(t+\zeta-\Delta t) d \zeta
\end{aligned}
$$

with the slippage given by

$$
c \Delta t=L \frac{\beta_{h}-\beta_{e}}{\beta_{e} \beta_{h}} .
$$

First, we assess the value of allowable slippage by the deconvolving equation (19), assuming that the shape of $\bar{I}_{\mathrm{e}}(t)$ repeats that of the hadron beam $I_{h}(t)$, e.g.,

$$
\frac{\bar{I}_{\mathrm{e}}(t)}{\bar{I}_{\mathrm{e}}(0)}=\frac{I_{h}(t)}{I_{h}(0)}=q(t) .
$$

Thus we require that

$$
\int_{0}^{\Delta t} I_{\mathrm{e}}(t-\Delta t+\zeta) d \zeta=I_{o} \cdot q(t)
$$

with a value of $I_{o}$ chosen to compensate the tune shift for the hadron in the center of the bunch. Any deviation of the e-bunch's shape from (21b) will result in error in the compensating tune for the hadrons. In Appendix D we show how to deconvolute

$$
\int_{0}^{\Delta t} g(t+\zeta) d \zeta=q(t) ; \quad I_{\mathrm{e}}(t-\Delta t)=I_{o} g(t),
$$

to get two simple independent solutions:

$$
\begin{aligned}
& g_{+}(t)=-\sum_{m=0}^{\infty} q^{\prime}(t+m \Delta t) ; \\
& g_{-}(t)=+\sum_{m=1}^{\infty} q^{\prime}(z-m \Delta t) .
\end{aligned}
$$

It is evident that a linear combination of the solutions (23)

$$
g_{\alpha}(t)=\alpha g_{+}(t)+(1-\alpha) g_{-}(t)
$$




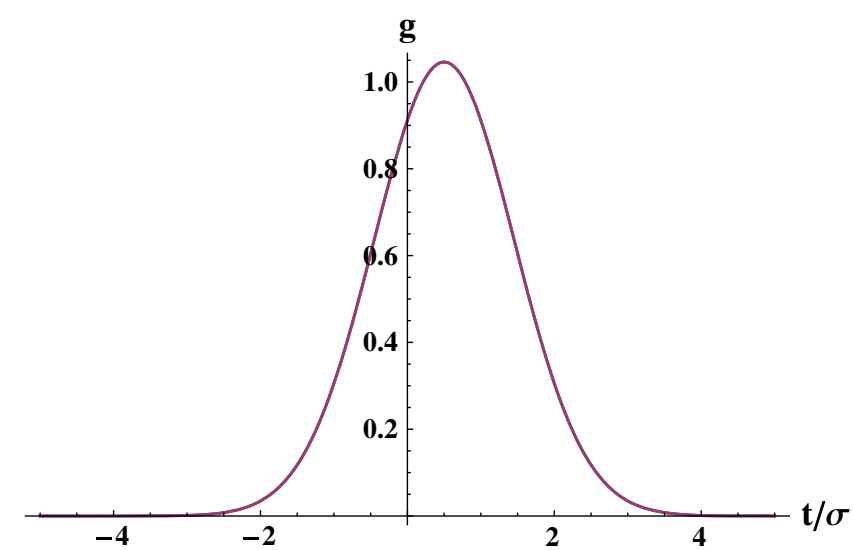

FIG. 2. Graphs of $g_{ \pm}(t)$ for deconvolution for Gaussian distribution (27) with $\tau=1$. We note that both functions $g_{ \pm}(t)$ are practically indistinguishable.

is a solution of (22). It is likely that $g_{1 / 2}=\left(g_{+}+g_{-}\right) / 2$ can be of practical interest.

We also show in Appendix D that for rather general physics assumptions, these functions converge to zero at one of the infinities:

$$
g_{+}(z)_{z \rightarrow+\infty} \rightarrow 0 ; \quad g_{-}(z)_{z \rightarrow-\infty} \rightarrow 0 .
$$

This is not necessarily true for the other sign. While these mathematical properties of the solutions mostly are of academic interest, there is an additional, very practical issue. By definition in Eq. (21a), $q(t)$ is a non-negative function: $q(t) \geq 0$. Similarly, the sign of the e-beam current is always negative, and $I_{\mathrm{e}}(t) \leq 0$. Thus, any practical deconvolution of (22) cannot change the sign, and choosing $I_{o}<0$ requires $g(t)$ be a positive function:

$$
g(t) \geq 0 .
$$

The natural parameter determining the behavior and "positivity" of $g_{ \pm}(t)$ is determined by the ratio between the slippage, $\Delta t$, and the rms length of the hadron bunch, $\sigma_{t}$,

$$
\tau=\frac{\Delta t}{\sigma_{t}} .
$$

In storage rings, the longitudinal distribution of the bunch frequently is described by a Gaussian function:

$$
q(t)=\exp \left[-\frac{t^{2}}{2 \sigma_{t}^{2}}\right]
$$

We studied this case in detail (see Appendix D) to answer the following questions: (a) At what values of $\tau$ do the deconvolution functions remain positively defined? (b) What error is accumulated in the convolution if we fit a reasonable positively defined function to approximate $g_{ \pm}(t)$ for large values of $\tau$ ?

The following shortly summarizes our findings. First, for $\tau \leq 1$, both $g_{ \pm}(t)$ solutions converge very well within the typical physical aperture (here we used $\pm 5 \mathrm{rms}$ bunch lengths). For $\tau \leq 1$, the deconvolutions $g_{ \pm}(t)$ are nearly identical (see Fig. 2) and are positively defined within the interval $t / \sigma_{t} \in\{-5,5\}$. For $\tau=0.5$, the difference between $g_{ \pm}(t)$ is within $\pm 10^{-15} g_{ \pm}(0)$ and likely is determined by the computer's accuracy. For $\tau=1$, the difference between $g_{ \pm}(t)$ is less than $\pm 10^{-7} g_{ \pm}(0)$ and $g_{ \pm}\left(5 \sigma_{t}\right) \cong 1.8 \times 10^{-5}, g_{ \pm}\left(-5 \sigma_{t}\right) \cong 9.5 \times 10^{-8}$. This simply means that, for practical purposes with $\tau \leq 1$, the compensating error will not be defined by the deconvolution function, but by other practical means.

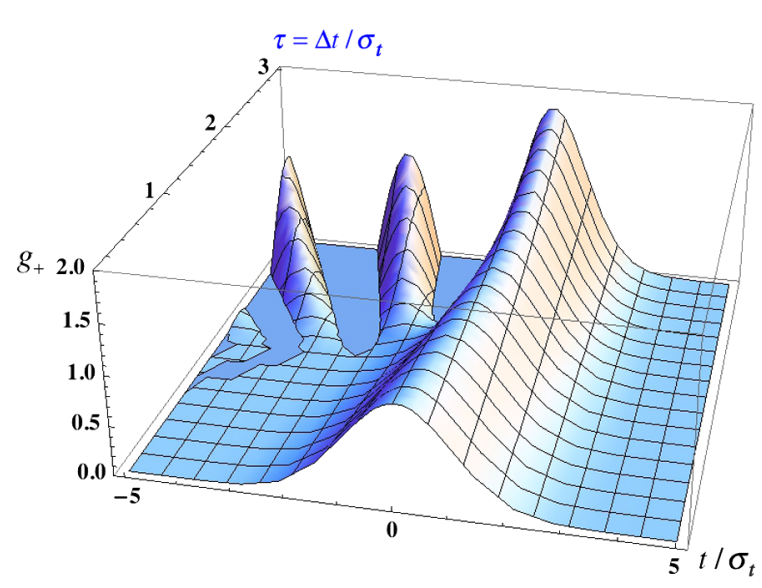

(a)

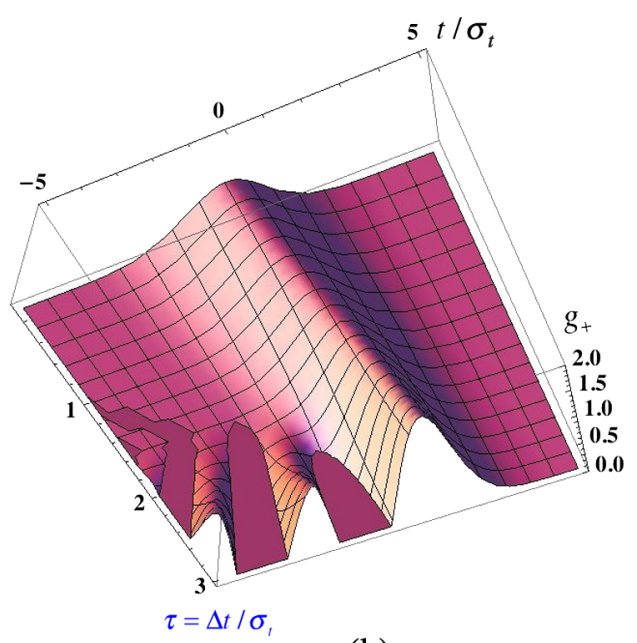

(b)

FIG. 3. 3D graphs of the deconvolution function $g_{+}$for Gaussian distribution (27) as a function of the time and slippage. Part (a) is the top view with the horizontal axis being $t / \sigma_{t}\{-5.5\}$, the vertical axis being $g_{+}(t)$, whilst the third axis used for the slippage $\tau=\Delta t / \sigma_{t} \in\{0,3\}$. The vertical axis is clipped at zero to show where $g_{+}$becomes negative. (b) The same graph seen from the bottom to illustrate the areas where $g_{+}<0$. 


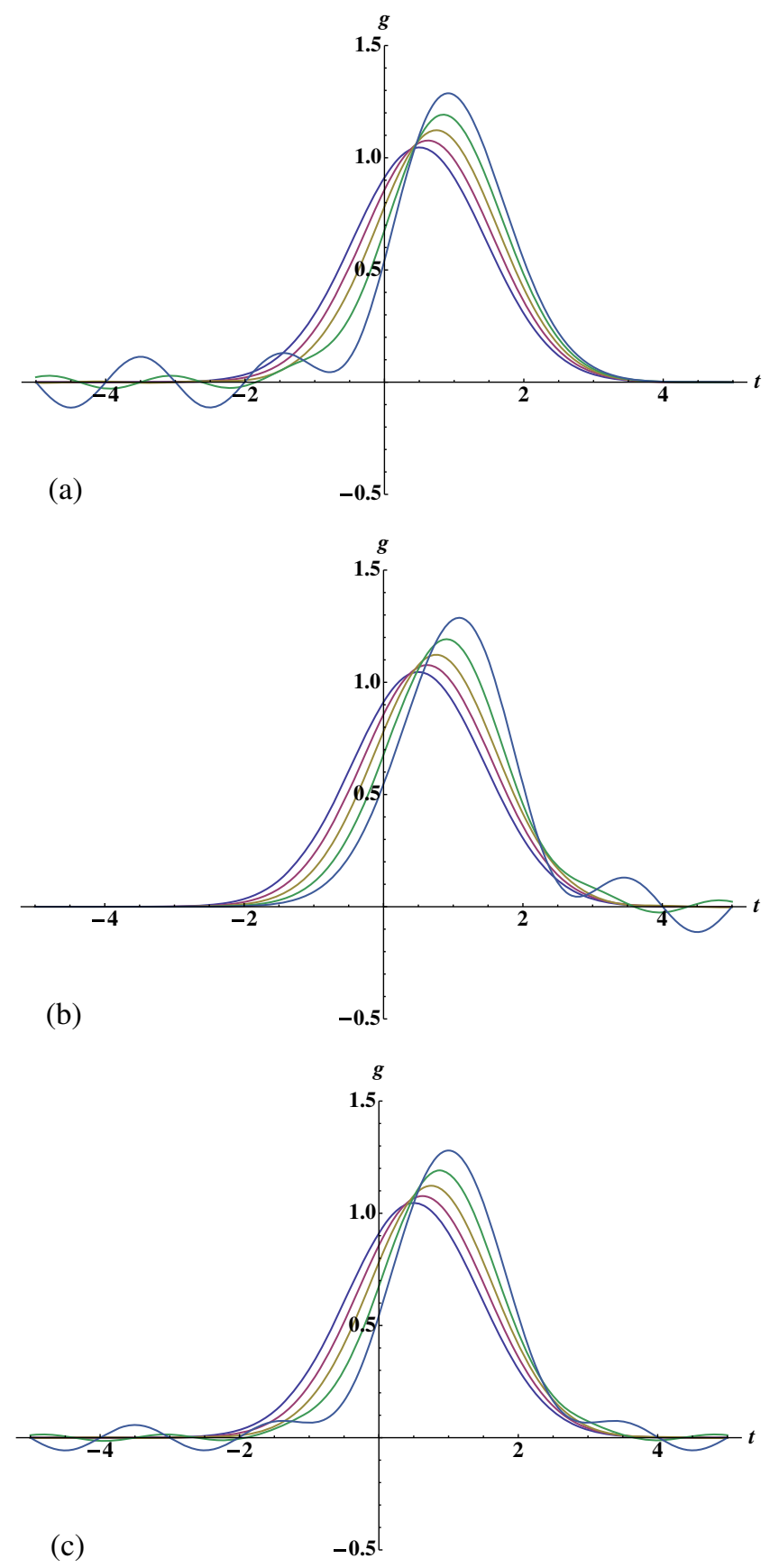

FIG. 4. 3D graphs of $g_{+}$(a), $g_{-}$(b), and $g_{1 / 2}$ (c) deconvolution functions for a Gaussian distribution (27) at function of the time for four values of $\tau=\Delta t / \sigma_{t}: 1-$ dark blue, 1.25-magenta, 1.5-yellow/grey, 1.75-green, and 2-light blue.

Second, for values of $\tau$ exceeding unity, the situation changes rather rapidly and when $\tau>1.75$ there are very well-defined oscillating tails with amplitudes comparable to that of the central peak. Naturally, both $g_{ \pm}(t)$ no longer are positively defined. Figures 3 and 4 illustrate these features.

The practical conclusion from these studies is that $\tau=1.5$ is a natural boundary, where a $g_{1 / 2}$ deconvolution

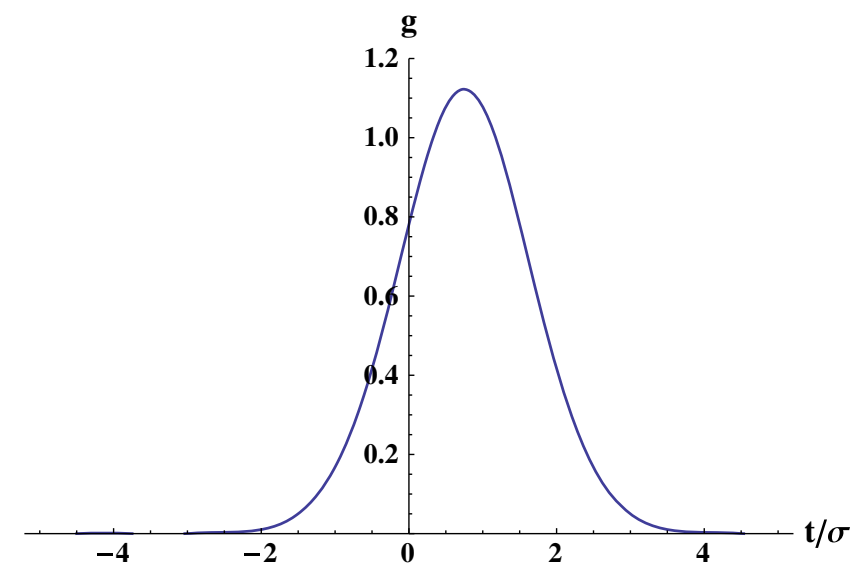

FIG. 5. Clipped graph of $g_{1 / 2}$ for a case of $\tau=1.5$. Keeping the shape of $g_{1 / 2}$ from $t=-3 \sigma_{t}$ to $t=4 \sigma_{t}$ assures a nearly perfect convolution (22).

(see Fig. 5) works very well. It provides a relative convolution error of less than $10^{-3}$ (to be exact it is limited to about $5 \times 10^{-4}$ ) and, therefore, would not represent practical limit to accuracy. For example, the bunch-tobunch intensity variation in a hadron beam most likely will exceed the relative level of $10^{-3}$.

As shown in Appendix D, even for $\tau=2$ we could find a positive function (by fitting $g$ ) that could compensate $95 \%$ of the tune spread. Nevertheless, for the rest of this paper we use $\tau=1.5$ as a practical limit for slippage.

Since we have proven that for a smooth Gaussian-like longitudinal distribution of the hadron bunch we nearly perfectly can compensate the space-charge-induced tune spread, the next question is what electron beam current is required to achieve this.

In Appendices $\mathrm{A}, \mathrm{B}$, and $\mathrm{C}$ we discuss issues related to the transverse matching of the nonlinear tune shift induced by the space charge in the ring and that induced by the electron beam in the compensator. Here, for simplicity, we further consider the space-charge effects for a round beam, and set $f_{x, y}=1$ in Eq. (2). ${ }^{6}$ Then, the space-charge tune shift accumulated by hadrons along the ring's circumference, $\mathrm{C}$, should be compensated by that accumulated in the interaction with electrons:

$$
\begin{aligned}
\delta Q_{\mathrm{sc}} & =-\frac{C}{4 \pi \varepsilon} \frac{1}{\beta_{h}{ }^{3} \gamma_{h}{ }^{3}} \frac{Z r_{p}}{A} \cdot \frac{I_{h}}{e c}=-\delta Q_{\mathrm{sc} e} \\
& =\frac{L_{c}}{8 \pi \varepsilon} \frac{1}{\beta_{h} \gamma_{h}} \frac{1-\beta_{e} \beta_{h}}{\beta_{e}} \frac{Z r_{p}}{A} \cdot \frac{\bar{I}_{e}}{e c} .
\end{aligned}
$$

\footnotetext{
${ }^{6}$ Compensation criteria do not depend on the details of the transverse matching. The latter is required to compensate correctly space-charge tune spreads in horizontal and vertical directions, as well as to approximate the dependencies of the space-charge tune shift on the transverse actions, $I_{x, y}$.
} 
Thus, the requirement for the compensating beam current,

$$
\begin{aligned}
\frac{L_{c}}{\beta_{e}} \bar{I}_{e} \cdot\left(1-\beta_{e} \beta_{h}\right) & =I_{h} C \frac{\left(1-\beta_{h}^{2}\right)}{\beta_{h}} \\
\frac{\bar{I}_{e}}{I_{h}} & =-\frac{C}{L_{c}} \frac{\beta_{e}}{\beta_{h}} \frac{\left(1-\beta_{h}^{2}\right)}{\left(1-\beta_{e} \beta_{h}\right)},
\end{aligned}
$$

depends on the length of the compensator section, $L_{c}$, that could be limited either by the allowable slippage, as we discussed above, $c \Delta t \leq 1.5 \sigma_{z} / \beta_{h}$,

$$
L_{\max } \leq c \Delta t_{\max } \frac{\beta_{e} \beta_{h}}{\beta_{h}-\beta_{e}} \approx \frac{1.5 \sigma_{z} \beta_{e}}{\beta_{h}-\beta_{e}},
$$

or by the practical limitations of the accelerator. Combined with the limitation on the slippage, this gives the following:

$$
\left|\frac{\bar{I}_{e}}{I_{h}}\right|=\frac{C}{\gamma_{h}^{2} \beta_{h}^{2}\left(1-\beta_{e} \beta_{h}\right)} \max \left\{\frac{\left|\beta_{e}-\beta_{h}\right|}{\Delta t c}, \frac{\beta_{e} \beta_{h}}{L_{c}}\right\} .
$$

As we discussed in previous sections, there is no benefit in having $\beta_{e} \geq \beta_{h}$. Hence, for $\beta_{e}<\beta_{h}$, Eq. (23) becomes

$\left|\frac{\bar{I}_{e}}{I_{h}}\right|=\frac{C}{\gamma_{h}^{2} \beta_{h}^{2}} \max \left\{\frac{\beta_{h}-\beta_{e}}{c \Delta t\left(1-\beta_{e} \beta_{h}\right)}, \frac{\beta_{e} \beta_{h}}{L_{c}\left(1-\beta_{e} \beta_{h}\right)}\right\}$.

To minimize the required electron beam current, we can find the minimum of the right-hand side in Eq. (32). We note that $\frac{\beta_{h}-\beta_{e}}{1-\beta_{e} \beta_{h}}=\beta_{h} \frac{1-\beta_{e} / \beta_{h}}{1-\beta_{e} \beta_{h}}$ monotonically decreases and $\frac{\beta_{h} \beta_{e}}{1-\beta_{e} \beta_{h}}$ monotonically increases as a function of $\beta_{e}$ at the interval $0 \leq \beta_{e} \leq \beta_{h}$. Thus the minimum in Eq. (32) is reached at

$$
\beta_{e}=\beta_{c}=\frac{\beta_{h}}{1+\beta_{h} \frac{c \Delta t}{L_{c}}} .
$$

This yields a simple expression for the required compensating current:

$$
\frac{\bar{I}_{e}}{I_{h}}=-\frac{C}{L_{c}+\gamma_{h}^{2} \beta_{h} \cdot c \Delta t} .
$$

In a case of more than one compensator $N_{c} \geq 1$, the required e-beam current can be reduced proportionally:

$$
\frac{\bar{I}_{e}}{I_{h}} \cong-\frac{1}{N_{c}} \frac{C}{L_{c}+\gamma_{h}^{2} \beta_{h} \cdot c \Delta t} .
$$

Having more than one compensator may offer additional advantages; it will distribute compensation around the ring. The latter will bring the compensators closer to the source and naturally will provide a more stable beam (see discussion at the end of the paper).

According to Eq. (35) using multiple compensators with the given total length of

$$
L=N_{c} L_{c}=\eta C,
$$

the required e-beam would be

$$
\frac{\bar{I}_{e}}{I_{h}} \cong-\frac{1}{\eta+N_{c} \cdot \gamma_{h}^{2} \beta_{h} \cdot \frac{c \Delta t}{C}} .
$$

Thus, it is beneficial to split compensation length into as many as practically manageable compensators. As follows from (33), this also will lower the optimal energy of the electron beam.

Such splitting will not necessarily lead to an increase of the electron beam sources and collector. Hadron storage rings usually operate with many hadrons bunches. For example, RHIC (and the future eRHIC) operate with 120 hadron bunches. Figure 6 shows a scheme in which one electron beam source can serve several space-charge compensators. This scheme is especially effective when using a low-energy electron beam moving with nonrelativistic velocities. In following section, we will discuss what limits the minimal length of the compensator.

\section{EFFECTS ON THE ELECTRON BEAM}

It is well known that hadron beam affects the propagation and dynamics of the electron beam. There is an economical value in using low-energy electron beams. However, such a

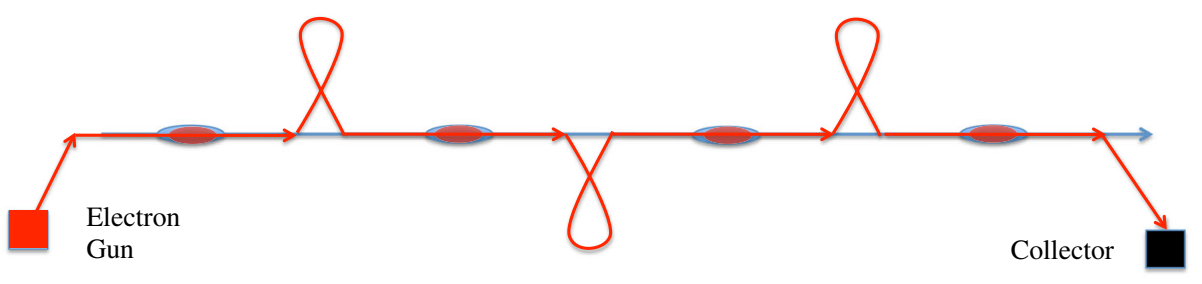

FIG. 6. A sketch of one electron source supporting four SC compensating sections. Each electron bunch (red) merges and copropagates with the hadron beam in the first straight section. Then it is sent through a delay loop to merge with the following hadron bunch. The process is then repeated as needed (3 times in this figure). 
beam would be highly susceptible to space-charge forces induced by itself as well as by the hadron beam. The solution for transporting such beams and accurately controlling their distribution is well known: that of a magnetized electron beam transported by a solenoidal field [29-40]. Such transport assures both the stability of the electron beam (and therefore, the hadron beam) as well as the control of the beam's size by changing the value of the solenoidal field. It provides focusing needed to counteract destructive space-charge forces.

For example, the stability of the interacting electron and hadron beams will be similar to that in head-on collisions using electron lens [40-44]. As shown in [45], using a strong longitudinal magnetic field plays an important role in maintaining the stability of these interacting beams.

If solenoidal transport is used, the main limiting factor for the compensator length will be the finite radius of curvature of the bends. At each bend, the hadron experiences the field from the bent electron beam, which has a different transverse structure from what we are compensating. Thus, the consequence of the end effects must be controlled, and we can formulate an addition limitation on the compensator's length:

$$
L_{c} \gg \text { bend radius. }
$$

In practice, this means that the typical lengths of a compensator should be a few meters and not a few centimeters.

\section{EXAMPLES}

We now consider a case a hadron beam in eRHIC with $\gamma_{h}=250$, an rms bunch length of $\sigma_{z}=8 \mathrm{~cm}$ and the compensator length of $3 \mathrm{~m}$. We assume that that slippage is equal to a $1.5 \mathrm{rms}$ bunch length, e.g., $c \Delta t=12 \mathrm{~cm}$. Equation (33) yields the optimum $\beta_{e} \cong 0.961$, and the optimum energy of the electron beam of $1.86 \mathrm{MeV}$ (kinetic energy of $1.35 \mathrm{MeV}$ ). The required electron beam current for a single compensator should be

$$
\bar{I}_{e} \cong-0.51 \cdot I_{h} .
$$

In a high-luminosity eRHIC, the expected proton intensity is $3 \times 10^{11}$ per bunch with the peak proton beam current of $\sim 72 \mathrm{~A}$. Hence, the required e-beam peak current $\sim 37 \mathrm{~A}$ is reasonable. For a $\gamma_{h}=50$, the required e-beam current would grow nearly 12.6-fold to about 900 A, making such compensation in a single compensator a very challenging task.

Hence, since the length of the compensation section is only $3 \mathrm{~m}$, while each of six eRHIC IR straight sections is $200 \mathrm{~m}$ long, we can consider multiple compensating sections to reduce the required e-beam peak current to tens of amps.

Compensating for space-charge effects also could be required for a low-energy scan in RHIC in search of the critical point in the QCD phase diagram [46-50]. For such a scan the relativistic $\gamma$ can be as low as 2.7 [51], but bunch intensity in such an operation is significantly lower than in eRHIC and the bunch length is much longer [52]. Tables I and II give a sample of hadron beams' energies, their parameters, and possible parameters for space-charge compensation.

Parameters of the compensators presented in the tables are not necessarily optimized for performance or cost. What is important is that the energy of an electron beam required for space-charge compensation for RHIC's low energy scan is in the $\sim 10 \mathrm{KeV}$ range (this was the reason for quoting kinetic energy in the table). It is related directly to using long bunches with an rms duration of a few meters. Such long bunches allow a longer slippage, and, therefore, operating e-beams with $\beta_{e} \sim 0.2-0.25$. This also means that the electrons are rather slow moving, and that the loops shown in Fig. 6 are 4-5-fold shorter than would be required for relativistic electrons.

In contrast, eRHIC will use short bunches of hadrons with rms length of few centimeters that results in a modest but relativistic energy of the electrons $\sim 1.35 \mathrm{MeV}$ with $\beta_{e} \cong 0.96$.

These simple examples show the ability of this concept to compensate the space-charge-induced tune spread with a compensator of reasonable length, and of a reasonable current.

\section{DISCUSSION AND CONCLUSIONS}

In this paper we presented a novel method of compensating space-charge-induced tune spread in bunched hadron beams. In principle, we showed that it is possible to compensate for both the tune shift and the tune spread with significant accuracy. We consider that, with a proper design and simulations, we can compensate for up to $99 \%$ of the space-charge-induced tune spread.

It is natural to question what would be the ultimate space-charge-induced tune spread that can be sustained in a storage ring. Unfortunately there is no simple direct answer. It is well known that space-charge-induced resonances can make hadron beam unstable [53-55]. Thus, the beam's dynamics in the presence of the compensators should be simulated using an appropriate code (for example [56-60]).

One important conclusion from such simulations (for the bunch-average tune shift compensation [56-59]) is that spreading the compensators around the ring allows us to achieve (at least in a simulation) larger beam intensities. A frequently observed phenomenon is that local compensation for nonlinear effects in beam dynamics is the preferable solution, e.g., placing the compensation as close as possible to the source.

Hence, we believe that having multiple tune spread compensators distributed around the ring would result in a better compensation and beam stability. For example, the strength and the locations of the compensators can be 
chosen to eliminate the most dangerous resonant driving terms.

This said, further detailed theoretical and numerical studies are required to find the limits of the proposed compensation scheme: what is the maximum space-charge tune shift/spread that can be compensated before the dynamic aperture of the machine collapses? or before the beam becomes unstable? $[61,62]$.

In contrast with traditional space-charge compensating schemes, we proposed the method of compensating not only the tune shift but also the entire tune spread. We expect that this method would stabilize the intense hadron beam in sub-TeVs and in low-energy storage rings. Electron beams with required quality, energy, and intensity either are readily available, or are being considered for future accelerators [63-66]. They could be used for the proposed compensator for space-charge effects.

We believe that using multiple compensators located in multiple straight sections of the storage ring (e.g., having them as close as possible to the source) would be required for compensating strong space-charge effects. Their locations (e.g., their strengths and betatron phases) have to be properly chosen to eliminate dangerous driving terms.

Finally, the main idea of this paper is based on the observation that for comoving electron and hadron beams the slippage is proportional to the difference between their velocities $L \cdot\left(\beta_{h}-\beta_{e}\right) / \beta_{e}$, while the reduction of the space-charge force is proportional to $\sim \gamma_{e}^{-2}+\gamma_{h}^{-2}$. It means that our proposed scheme strongly enhances compensation for relativist and ultrarelativistic hadron beams with $\gamma_{h} \gg 1$.

TABLE I. Examples of possible space-charge compensator schemes for various modes of operating the highluminosity eRHIC.

\begin{tabular}{lcccc}
\hline \hline Parameter & & & & \\
\hline Hadron beam & $\mathrm{p}$ & $\mathrm{p}$ & ${ }^{79} \mathrm{Au}^{197}$ & ${ }^{79} \mathrm{Au}^{197}$ \\
Energy, GeV/u & 250 & 100 & 100 & 50 \\
Number of particles & $3 \times 10^{11}$ & $3 \times 10^{11}$ & $3 \times 10^{9}$ & $3 \times 10^{9}$ \\
rms bunch length, m & 0.08 & 0.08 & 0.08 & 0.08 \\
Normalized emittance, m rad & $2 \times 10^{-7}$ & $2 \times 10^{-7}$ & $2 \times 10^{-7}$ & $2 \times 10^{-7}$ \\
$\Delta Q_{\text {sc }}$ & 0.05 & 0.31 & 0.1 & 0.40 \\
Peak current, A & 72 & 72 & 58 & 58 \\
Electron beam & & & 3 & 3 \\
Compensator length, m & 3 & 3 & 1.35 & 1.35 \\
Kinetic energy, MeV & 1.35 & 1.35 & 12 & 24 \\
Number of compensators & 3 & 12 & 13.1 & 26 \\
Peak current, A & 10.6 & 16.6 & 36 & 74 \\
Total compensators length, m & 9 & 36 & & 35 \\
\hline \hline
\end{tabular}

TABLE II. Examples of possible space-charge compensator schemes for various modes of low energy RHIC operations.

\begin{tabular}{lcccc}
\hline \hline Parameter & & & & \\
\hline Hadron beam & ${ }^{79} \mathrm{Au}^{197}$ & ${ }^{79} \mathrm{Au}^{197}$ & ${ }^{79} \mathrm{Au}^{197}$ & ${ }^{79} \mathrm{Au}^{197}$ \\
Energy, GeV/u & 2.5 & 3.85 & 5.75 & 10 \\
Number of particles & $0.5 \times 10^{9}$ & $1.1 \times 10^{9}$ & $1.1 \times 10^{9}$ & $1.1 \times 10^{9}$ \\
rms bunch length, m & 3 & 3 & 2 & 2 \\
Norm. emittance, m rad & $1.5 \times 10^{-6}$ & $1.5 \times 10^{-6}$ & $1.5 \times 10^{-6}$ & $1.5 \times 10^{-6}$ \\
$\Delta Q_{\text {sc }}$ & 0.10 & 0.09 & 0.06 & 0.075 \\
Peak current, A & 0.25 & 0.55 & 0.82 & 0.83 \\
Electron beam & & & 1 & 1 \\
Compensator length, m & 1 & 1 & 16.5 & 16.5 \\
Kinetic energy, keV & 8.6 & 8.6 & 12 & 12 \\
Number of compensators & 12 & 12 & 2.4 & 0.77 \\
Peak current, A & 2.5 & 2.4 & 12 & 12 \\
Total compensators length, m & 12 & 12 & & 12 \\
\hline \hline
\end{tabular}

*Ion beam parameters are taken from [51,52], and we assume that the beam's transverse emittance is cooled by a factor of $2[67,68]$. 
What is also important, in our scheme the slippage can be controlled by varying $\beta_{h}-\beta_{e}<\beta_{h}$, while in the head-on collision scheme aka e-lenas it is proportional to $\beta_{h}+\beta_{e}>\beta_{h}$. The later can be beneficial also for hadron rings with modest energy $\gamma_{h}=2-10$. For lower energy hadron rings (synchrotrons) with $\gamma_{h} \sim 1$ operating very long bunches, the benefit of our scheme can be modest, when compared with the head-on collision scheme [24]. For example, for the $2.5 \mathrm{GeV} / \mathrm{u}$ ion beam proposed for the low energy RHIC operation (see column one in Table II), the copropagating of the electron beam reduces the slippage 1.5-fold compared with the head-on compensator.

Thus, for low energy hadron rings with $\gamma_{h} \sim 1$ operating beam with bunch length $\sim 10 \mathrm{~m}$ or longer, the degree of improvement provided by our scheme would be very modest.

For hadron rings with $\gamma_{h} \gg 1$ operating beams with bunch length $\sim 1 \mathrm{~m}$ or shorter, our scheme gives a significant advantage. It is likely the only scheme, which could do the job with attainable electron beam parameters.

Let us review the case of eRHIC beam with energy $250 \mathrm{GeV}$ and rms bunch length $0.08 \mathrm{~m}$ (see column one in Table I). Our scheme increases the compensation strength of the electron beam 2600-fold, compared with "electron-cooling-like" scheme $\left(\gamma_{e}=\gamma_{h}\right)$. By doing so, it reduces the required e-beam peak current to tens of amps, from hundreds of $\mathrm{kA}$ required otherwise. When compared with the head-on collision, our scheme reduces the slippage between two beams 52-fold. Hence, a head-on scheme using relativistic electron beam must reduce the interaction length below $6 \mathrm{~cm}$. If a typical $10-\mathrm{keV}$ electron lens is used, the interaction length must be reduced even further, below $2 \mathrm{~cm}$. Providing such short interaction regions will be a very difficult, if not impossible, task.

Thus we conclude that our novel scheme provides a practical method of compensating the tune shift and the tune spread induced by space charge in bunched hadron beams.

\section{ACKNOWLEDGMENTS}

The authors are indebted to Alexey Fedotov (BNL) for indispensable help in directing us toward the most recent studies and publications on space-charge related effects. We also appreciate in-depth discussions with Alexei of the relevant formulas. We are grateful to Ilan Ben-Zvi and Thomas Roser (both BNL) for their interest in our approach and thoughtful comments and suggestions. We would like to thank Vladimir Shiltsev (FNAL) for providing us with the latest copy of his presentation on various space-charge compensation ideas, such as the electron lens and plasma column. Our special thanks go to Alexander Pikin (BNL), who shared with us his deep knowledge of the beam parameters and shapes attainable with low energy electron beams. Work is supported by Brookhaven Science
Associates, LLC under Contract No. DE-AC0298CH10886 with the U.S. Department of Energy.

\section{APPENDIX A: SPACE-CHARGE-INDUCED TUNE SPREAD}

There are multiple ways of deriving the space-chargeinduced tune shift and tune spread for a relativistic hadron beam. We refer readers to specialized papers on the topic $[69,70]$ and the references therein. There is no practical limit to which one can complicate the space-charge problem by adding the effects of the surrounding environment [71], nontrivial beam distributions, and an arbitrary coupling between 3 degrees of freedom. While interesting in general, heavy mathematical calculations could obscure the main idea of this paper, i.e., describing the novel compensation method for the space-charge-induced tune spread.

Hence, we will focus on a case of uncoupled transverse motion with a simple Gaussian transverse and longitudinal density distribution in the bunch propagating in a vacuum:

$$
\begin{aligned}
& f(x, y, s, \tau=c t) \\
& \quad=\frac{N_{o}}{(2 \pi)^{3 / 2} \sigma_{x} \sigma_{y} \sigma_{z}} \exp \left(-\frac{x^{2}}{2 \sigma_{x}^{2}}-\frac{y^{2}}{2 \sigma_{y}^{2}}-\frac{\left(s-\beta_{o} \tau\right)^{2}}{2 \sigma_{z}^{2}}\right),
\end{aligned}
$$

where $N_{o}$ is the number of particles in the bunch, $\beta_{o} \equiv\left(1-\gamma_{o}^{-2}\right)^{1 / 2}=\mathrm{v}_{o} / c$ is the beam's longitudinal velocity, $\tau=c t$ and $c$ is the speed of the light. Trivial Lorentz transformation gives us the distribution (A1) in the comoving frame:

$$
\begin{aligned}
\bar{f}(x, y, \bar{z}) & =\frac{N_{o}}{(2 \pi)^{3 / 2} \gamma_{o} \sigma_{x} \sigma_{y} \sigma_{z}} \exp \left(-\frac{x^{2}}{2 \sigma_{x}^{2}}-\frac{y^{2}}{2 \sigma_{y}^{2}}-\frac{\bar{z}^{2}}{2 \bar{\sigma}_{z}^{2}}\right) ; \\
\bar{z} & =\gamma_{o}\left(s-\beta_{o} \tau\right) ; \quad \bar{\sigma}_{z}=\gamma_{o} \sigma_{z} .
\end{aligned}
$$

The charge density, $\rho$, differs from the particle density by the multiplier $e Z$. We assume that, in the comoving frame, the scalar potential is nearly time impendent, viz., it evolves only with a change of the particles' distribution. Naturally, since there is no current in the comoving frame, the vector potential from space charge is zero. Now, we need to solve only the stationary Poisson equation ${ }^{7}$ :

$$
\Delta \bar{\varphi}(\vec{r})=-4 \pi \rho(\vec{r})
$$

Following [72], we can derive the scalar potential using well-known equalities $[73,74]$ :

\footnotetext{
${ }^{7} \mathrm{We}$ assume here that in the comoving frame speed of the particles is much smaller than the speed of light, and that the formula for a static scalar potential, $\Delta \varphi=-4 \pi \rho$, is applicable.
} 


$$
\bar{\varphi}(\vec{r})=\int \frac{\rho(\vec{\zeta})}{|\vec{r}-\vec{\zeta}|} d \vec{\zeta}^{3} ; \quad \frac{1}{|\vec{r}-\vec{\zeta}|} \equiv \frac{2}{\sqrt{\pi}} \int_{0}^{\infty} \exp \left(-u^{2}|\vec{r}-\vec{\zeta}|^{2}\right) d u
$$

and rewrite it using $q=1 / u^{2}$, as

$$
\bar{\varphi}(\vec{r})=\frac{1}{\sqrt{\pi}} \int_{0}^{\infty} \int q^{-3 / 2} \rho(\vec{\zeta}) \exp \left(-\frac{|\vec{r}-\vec{\zeta}|^{2}}{q}\right) d q d \vec{\zeta}^{3}
$$

Substituting the charge density using the particle distributions (A2),

$\bar{\varphi}(x, y, \bar{z})=\frac{e Z}{\sqrt{\pi}} \frac{N_{o}}{(2 \pi)^{3 / 2} \gamma_{o} \sigma_{x} \sigma_{y} \sigma_{z}} \int_{0}^{\infty} \frac{d q}{q^{3 / 2}} \int d \xi d \eta d \zeta \cdot \exp \left(-\frac{(x-\xi)^{2}+(y-\eta)^{2}+(\bar{z}-\zeta)^{2}}{q}-\frac{\xi^{2}}{2 \sigma_{x}^{2}}-\frac{\eta^{2}}{2 \sigma_{y}^{2}}-\frac{\zeta^{2}}{2 \bar{\sigma}_{z}^{2}}\right)$

and taking three trivial integrals, as indicated below,

$$
\begin{aligned}
\frac{(x-\xi)^{2}}{q}+\frac{\xi^{2}}{2 \sigma_{x}^{2}} & =\frac{q+2 \sigma_{x}^{2}}{2 \sigma_{x}^{2} q}\left(\xi-x \frac{2 \sigma_{x}^{2}}{q+2 \sigma_{x}^{2}}\right)^{2}+\frac{x^{2}}{q+2 \sigma_{x}^{2}} \\
\int_{-\infty}^{\infty} d \xi \exp \left(-\frac{(x-\xi)^{2}}{q}-\frac{\xi^{2}}{2 \sigma_{x}^{2}}\right) & =\sigma_{x} \sqrt{\frac{2 \pi q}{q+2 \sigma_{x}^{2}}} \exp \left(-\frac{x^{2}}{q+2 \sigma_{x}^{2}}\right)
\end{aligned}
$$

we derive the final expression for a scalar potential:

$$
\bar{\varphi}(x, y, \bar{z})=\varphi_{o}+\frac{e Z}{\sqrt{\pi}} N_{o} \cdot \int_{0}^{\infty} d q \frac{\exp \left(-\frac{x^{2}}{q+2 \sigma_{x}^{2}}-\frac{y^{2}}{q+2 \sigma_{y}^{2}}-\frac{\bar{z}^{2}}{q+2 \bar{\sigma}_{z}^{2}}\right)}{\sqrt{\left(q+2 \sigma_{x}{ }^{2}\right)\left(q+2 \sigma_{y}{ }^{2}\right)\left(q+2 \bar{\sigma}_{z}^{2}\right)}} .
$$

Since in the comoving frame only the time component of the 4-vector potential, e.g., $\bar{\varphi}$, is nonzero, the Lorenz transformation back into the laboratory frame is trivial:

$$
\left[\varphi_{\mathrm{sc}}(x, y, s, \tau), \vec{A}_{\mathrm{sc}}(x, y, s, \tau)\right]_{\mathrm{lab}}=\gamma_{o}\left(1, \beta_{o} \hat{s}\right) \cdot \bar{\varphi}\left[x, y, \gamma\left(s-\beta_{o} \tau\right)\right]
$$

where $\hat{s}$ is the unit vector along the beam propagation. Equation (A5) can be directly added into the Canonical accelerator Hamiltonian $[75,76]$,

$$
h^{*}=-(1+K x) \sqrt{\frac{\left(H-e \varphi+e \varphi_{\mathrm{sc}}\right)^{2}}{c^{2}}-m^{2} c^{2}-\left(P_{x}-\frac{e}{c} A_{x}\right)^{2}-\left(P_{y}-\frac{e}{c} A_{y}\right)^{2}}-\frac{e}{c}\left(A_{2}+A_{c s}\right),
$$

where we assumed a flat ring reference orbit (i.e., the absence of orbit torsion [75]).

While it is possible to proceed further using the Hamiltonian (A6), for most practical cases, we can use significant simplifications. First, in all practical hadron storage rings, the bunch length is significantly larger than the transverse beam sizes, e.g., $\sigma_{z} \gg \sigma_{x, y}$. For example, in eRHIC, the hadron beam rms bunch length will be $\sim 50 \mathrm{~mm}$, while the transverse beam size will be $\sim 0.2 \mathrm{~mm}$. With a further $\bar{\sigma}_{z}=\gamma_{o} \sigma_{z}$ boost in the comoving frame, this size asymmetry becomes overwhelming, e.g.,

$$
\bar{\sigma}_{z} \ggg \sigma_{x, y} .
$$


In the case of eRHIC case, we would have $\bar{\sigma}_{z} / \sigma_{x, y}>10^{4}$. This asymmetry allows using a two-dimensional expression for the scalar potential. The easiest way is using the $\bar{\sigma}_{z}{ }^{2} \rightarrow \infty$ limit in (A4), but it also can be done directly [77] $]^{8}$ :

$$
\bar{\varphi}(x, y, \bar{z})=\frac{e Z N_{o}}{\sqrt{2 \pi} \bar{\sigma}_{z}} \mathrm{e}^{-\frac{\bar{z}^{2}}{2 \bar{\sigma}_{z}^{2}}} \cdot \int_{0}^{\infty} d q \frac{\exp \left(-\frac{x^{2}}{q+2 \sigma_{x}^{2}}-\frac{y^{2}}{q+2 \sigma_{y}^{2}}\right)}{\sqrt{\left(q+2 \sigma_{x}^{2}\right)\left(q+2 \sigma_{y}^{2}\right)}} .
$$

Following [69] to keep potential finite on the beam axis $(x=y=0)$ one should subtract unity for the exponent in the nominator: $\exp \left(-\frac{x^{2}}{q+2 \sigma_{x}^{2}}-\frac{y^{2}}{q+2 \sigma_{y}^{2}}\right)-1$. We implicitly assume such subtraction where it is needed.

Using substitutions $u(s)=\sigma_{x}(s) / \sigma_{y}(s)$ and $q=2 \vartheta \sigma_{x} \sigma_{y}$. Equation (A7) can be rewritten with a dimensionless integral:

$$
\bar{\varphi}(x, y, \bar{z})=\frac{e Z N_{o}}{\sqrt{2 \pi} \bar{\sigma}_{z}} \mathrm{e}^{-\frac{\bar{z}^{2}}{2 \bar{\sigma}_{z}^{2}}} \cdot \int_{0}^{\infty} d \vartheta \frac{\exp \left(-\frac{x^{2} / \sigma_{x}^{2}}{2(1+\vartheta / u)}-\frac{y^{2} / \sigma_{y}^{2}}{2(1+\vartheta u)}\right)}{\sqrt{(1+\vartheta / u)(1+\vartheta u)}}
$$

The near-the-axis expansion of Eq. (A7) can be found written in analytical form using an identity $a(a+b) \int_{0}^{\infty} d q / \sqrt{\left(q+2 a^{2}\right)^{3}\left(q+2 b^{2}\right)}=1$ :

$$
\bar{\varphi}(x, y, \bar{z})=-\frac{e Z N_{o}}{\sqrt{2 \pi} \bar{\sigma}_{z}} \mathrm{e}^{-\frac{\bar{z}^{2}}{2 \bar{\sigma}_{z}^{2}}} \cdot \frac{1}{\sigma_{x}+\sigma_{y}}\left(\frac{x^{2}}{\sigma_{x}}+\frac{y^{2}}{\sigma_{y}}\right)+O^{4}
$$

The next useful approximation, which is very common in the accelerator literature, originates from the fact that synchrotron oscillations in hadron rings are much slower than the transverse (betatron) oscillation. It allows us to consider both the particle's longitudinal position inside the bunch as well as its relative energy deviation, $\delta \equiv E / E_{o}-1$, as slow varying. In this approximation, the transverse accelerator Hamiltonian (with the dimensionless variables $p_{x, y} \rightarrow p_{x, y} / p_{o}$ ) becomes $[75,76]$

\footnotetext{
${ }^{8}$ There is a direct way of doing deriving (A7) following [77]. By applying a Fourier transform $\iint . . \exp (\vec{k} \vec{r}) d x d y /(2 \pi)^{2}$ to $\Delta_{\perp} \varphi \approx-4 \pi \rho$; for $\bar{\sigma}_{z} \gg \sigma_{x, y}$ we obtain $\varphi(\vec{k})=4 \pi \rho(\vec{k}) / \vec{k}^{2}$. Using a familiar trick $\frac{1}{\vec{k}^{2}}=\int_{0}^{\infty} e^{-\vec{k}^{2} t} d t \equiv \frac{1}{4} \int_{0}^{\infty} e^{-\frac{\vec{k}_{t}^{2}}{4}} d t$ and scaling it by $1 / 4$ we get

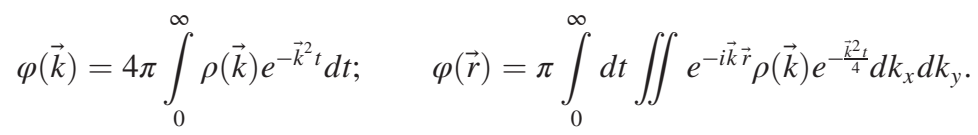

Then, for a long Gaussian bunch with linear density of $\rho_{o}(z)=e Z N \cdot e^{-\frac{z^{2}}{2 \sigma_{z}^{2}}} /\left(\sqrt{2 \pi} \bar{\sigma}_{z}\right)$.

$$
\rho=\rho_{o}(z) \frac{1}{2 \pi \sigma_{x} \sigma_{y}} e^{-\frac{x^{2}}{2 \sigma_{x}^{2}}-\frac{y^{2}}{2 \sigma_{y}^{2}}} ; \quad \rho(k)=\frac{1}{(2 \pi)^{2}} \cdot e^{-\frac{k_{x}^{2} \sigma_{x}^{2}}{2}-\frac{k_{y}^{2} \sigma_{y}^{2}}{2}}
$$

after trivial integration,

$$
\varphi(\vec{r})=\pi \rho_{o}(z) \frac{1}{(2 \pi)^{2}} \cdot \int_{0}^{\infty} d t \iint e^{-i \vec{k} \vec{r}} e^{-\frac{k_{x}^{2} \sigma_{x}^{2}}{2}-\frac{k_{y}^{2} \sigma_{y}^{2}}{2}} e^{-\frac{\vec{k}^{2} t}{4}} d k_{x} d k_{y} ; \quad \int_{-\infty}^{\infty} e^{-i k_{x} x} e^{-\frac{k_{x}^{2}\left(2 \sigma_{x}^{2}+t\right)}{4}} d k_{x}=\sqrt{\frac{4 \pi}{2 \sigma_{x}^{2}+t}} e^{-\frac{x^{2}}{2 \sigma_{x}^{2}+t}} ;
$$

we get the desirable result identical to (A7):

$$
\varphi(\vec{r})=\frac{e Z N}{\sqrt{2 \pi} \bar{\sigma}_{z}} e^{-\frac{z^{2}}{2 \sigma_{z}^{2}}} \cdot \int_{0}^{\infty} \frac{e^{-\frac{x^{2}}{2 \sigma_{x}^{2}+t}-\frac{y^{2}}{2 \sigma_{y}^{2}+t}}}{\sqrt{\left(2 \sigma_{x}^{2}+t\right)\left(2 \sigma_{y}^{2}+t\right)}} d t .
$$




$$
\begin{gathered}
\tilde{h}=-(1+K x) \sqrt{1+\frac{2}{\beta_{o}}\left(\delta-\frac{Z e}{p_{o} c} \varphi_{\mathrm{sc}}\right)+\left(\delta-\frac{Z e}{p_{o} c} \varphi_{\mathrm{sc}}\right)^{2}-p_{x}^{2}-p_{y}^{2}}-\frac{Z e}{p c}\left(A_{s}+A_{\mathrm{sc}}\right) ; \\
{\left[\varphi_{\mathrm{sc}}(x, y, s, \tau), \vec{A}_{\mathrm{sc}}(x, y, s, \tau)\right]_{\mathrm{lab}}=\gamma_{o}\left(1,+\beta_{o} \hat{s}\right) \cdot \bar{\varphi}\left[x, y, \gamma\left(s-\beta_{o} \tau\right)\right],}
\end{gathered}
$$

where $K$ is the curvature of the reference orbit.

Assuming that space charge can be treated locally as a perturbation (e.g., $\left|\Delta Q_{\text {sc } x, y}\right| \ll Q_{x, y}$ ), we can expand the Hamiltonian keeping only the dominant space-charge term. Using (A5) and taking into account that $\gamma_{o}\left(1-\beta_{0}^{2}\right)=\gamma^{-1}$, we obtain

$$
\tilde{h}=\tilde{h}_{o}+\Delta \tilde{h}_{\mathrm{sc}} \cong-(1+K x) \sqrt{1+\frac{2 \delta}{\beta_{o}}+\delta^{2}-\vec{p}_{\perp}^{2}}-\frac{Z e}{p c} A_{s}+\left\{\frac{1+K x}{1+\delta / \beta_{o}}\right\} \cdot \frac{Z e}{\beta_{o} \gamma_{o} p_{o} c} \bar{\varphi}\left[x, y, \gamma\left(s-\beta_{o} \tau\right)\right] .
$$

Since we are interested in the main space-charge effects, keeping the term in angular brackets is unnecessary. ${ }^{9}$ In (A11), the third term represents space-charge effects:

$$
\Delta \tilde{h}_{\mathrm{sc}}=\frac{1}{\beta_{o}^{2} \gamma_{o}^{3}} \frac{Z^{2} N_{o} r_{c}}{\sqrt{2 \pi} \sigma_{z}} \mathrm{e}^{-\frac{\left(s-\beta_{o}\right)^{2}}{2 \sigma_{z}^{2}}} \cdot \int_{0}^{\infty} d \vartheta \frac{\exp \left(-\frac{x^{2} / \sigma_{x}^{2}}{2(1+\vartheta / u)}-\frac{y^{2} / \sigma_{y}^{2}}{2(1+\vartheta u)}\right)}{\sqrt{(1+\vartheta / u)(1+\vartheta u)}}
$$

where $r_{c}=e^{2} / m c^{2}$ is the classical particle's radius. Since we are using $Z e$ as the charge of the hadrons, we also should introduce the hadron's mass number, $A=m / m_{p}$ (with $A>1$ for ions), where $m_{p}$ is the mass of the proton: $r_{c}=r_{p} / A ; r_{p}=e^{2} / m_{p} c^{2}$.

\footnotetext{
${ }^{9}$ The multiplier $1 /\left(1+\delta / \beta_{o}\right)$ describing the chromaticity of the space-charge effects can be estimated as $\sigma_{\delta} \cdot \Delta Q_{\mathrm{sc}} \sim 10^{-3} \Delta Q_{\mathrm{sc}}$, while the multiplier $1+K x$ represents the lengthening of the trajectory, and can be estimated as $\alpha_{c} \sigma_{\delta} \cdot \Delta Q_{\mathrm{sc}} \sim 10^{-3} \Delta Q_{\mathrm{sc}} / \gamma_{t}^{2}$, where $\gamma_{t}$ is the relativistic factor at transition energy. Typically, $\gamma_{t} \sim Q_{x} \gg 1$. More accurate treatment requires averaging the perturbation of the Hamiltonian over the phases of the betatron oscillations and integrating it over the ring circumference, $C$. It gives us an effective one-turn variation of the average Hamiltonian [76]:
}

$$
\begin{aligned}
\Delta \tilde{h}_{\mathrm{sc}} & =\frac{1+K x}{\beta_{o}+\delta} \cdot \frac{e}{\gamma_{o} p_{o} c} \bar{\varphi}(x, y, \bar{z}) ; \quad h_{c s}\left(I_{x}, I_{y}, z, \delta\right)=\frac{e}{\gamma_{o} p_{o} c} \frac{1}{\beta_{o}+\delta} \int_{0}^{c} d s\langle(1+K x) \cdot \bar{\varphi}(x, y, \bar{z})\rangle_{\phi_{x, y}} ; \\
x & =\sqrt{2 \beta_{x}(s) I_{x}} \cos \left[\psi_{x}(s)+\phi_{x}\right]+D_{x} \delta ; \quad y=\sqrt{2 \beta_{y}(s) I_{y}} \cos \left[\psi_{y}(s)+\phi_{y}\right],
\end{aligned}
$$

where $I_{x}, I_{y}$ are the actions of the betatron oscillations, $\delta$ is the relative energy deviation of the hadron, and $\beta_{x, y}$ are the lattice functions. The action dependent tune shift than can be calculated as follows:

$$
\Delta Q_{\text {sc } x, y}=\frac{1}{2 \pi} \frac{\partial h_{c s}\left(I_{x}, I_{y}, z, \delta\right)}{\partial I_{x, y}}
$$

This formula covers all aspects of the space-charge tune shift, including its chromaticity. For on-energy particle, $\delta=0$,

$$
h_{c s}\left(I_{x}, I_{y}, z\right)=\int_{0}^{C} d s\left\langle\frac{1+K x}{\gamma_{o} \beta_{o}} \cdot \frac{e}{p_{o} c} \bar{\varphi}(x, y, \bar{z})\right\rangle_{\phi_{x, y}} ; \quad x=\sqrt{2 \beta_{x}(s) I_{x}} \cos \left[\psi_{x}(s)+\phi_{x}\right] ; \quad y=\sqrt{2 \beta_{y}(s) I_{y}} \cos \left[\psi_{y}(s)+\phi_{y}\right] .
$$

Since the potential is symmetric function $\varphi(-x, y, z)=\varphi(x, y, z)$, and $\langle x \bar{\varphi}(x, y, \bar{z})\rangle \equiv 0$. Hence, the relative strength of neglected terms would be $\sim 10^{-3}$. 
Near the axis expansion of Eq. (A12) is straightforward using (A9):

$$
\begin{aligned}
\Delta \tilde{h}_{\mathrm{sc} L}= & -\frac{1}{\beta_{o}^{2} \gamma_{o}^{3}} \frac{Z^{2} N_{o} r_{p}}{A \sqrt{2 \pi} \sigma_{z}} \mathrm{e}^{-\frac{\left(s-\beta_{o} \tau\right)^{2}}{2 \sigma_{z}}} \\
& \times\left(\frac{x^{2}}{\sigma_{x}\left(\sigma_{x}+\sigma_{y}\right)}+\frac{y^{2}}{\sigma_{y}\left(\sigma_{x}+\sigma_{y}\right)}\right) .
\end{aligned}
$$

The classical averaging method ${ }^{10}$ over the fast betatron oscillations $[75,76]$

$$
\begin{aligned}
\Delta h_{\mathrm{sc}}\left(I_{x}, I_{y}\right) & =\left\langle\Delta \tilde{h}_{\mathrm{sc}}(x, y)\right\rangle_{\phi_{x}, \phi_{y}} ; \\
x & =\sqrt{2 \beta_{x}(s) I_{x}} \cos \left[\psi_{x}(s)+\phi_{x}\right] ; \\
y & =\sqrt{2 \beta_{y}(s) I_{y}} \cos \left[\psi_{y}(s)+\phi_{y}\right],
\end{aligned}
$$

where $I_{x, y}$ and $\phi_{x, y}$ are the actions and phases of the betatron oscillations, allows us to find local variation of the betatron phases as derivatives over the corresponding action:

$$
\frac{d \phi_{x, y}}{d s}=\frac{\partial \Delta h_{\mathrm{sc}}\left(I_{x}, I_{y}, s\right)}{\partial I_{x, y}}
$$

Then, the tune shift is a simple integral over the ring circumference:

$$
\Delta Q_{\mathrm{sc} x, y}=\frac{1}{2 \pi} \int_{o}^{C} d s \frac{\partial \Delta h_{\mathrm{sc}}\left(I_{x}, I_{y}, s\right)}{\partial I_{x, y}} .
$$

Averaging (A12) over betatron phases is straightforward using the well-known expression:

$F(\xi)=\left\langle e^{-2 \xi \cos ^{2} \psi}\right\rangle_{\psi} \equiv \frac{1}{2 \pi} \int_{o}^{2 \pi} d \psi e^{-2 \xi \cos ^{2} \psi}=e^{-\xi} \cdot I_{o}(\xi)$,

where $I_{o}(\xi)$ is the modified Bessel function of the first kind [74]. Expressing the beam sizes through the lattice functions, $\beta_{x, y}$, and the geometric emittances $\varepsilon,{ }^{11}$ the transverse dispersion, $D$, and the rms energy spread, $\sigma_{\delta}$,

$\sigma_{x}^{2}(s)=\beta_{x}(s) \varepsilon_{x}+D_{x}^{2}(s) \sigma_{\delta}^{2} ; \quad \sigma_{y}^{2}(s)=\beta_{y}(s) \varepsilon_{y}$,

and using Eq. (A16) we get

\footnotetext{
${ }^{10}$ With averaging over the phases of the betatron oscillations defined as $(2 \pi)^{2}\langle f\rangle_{\phi_{x}, \phi_{y}} \equiv \int_{0}^{2 \pi} \int_{0}^{2 \pi} f d \phi_{x} d \phi_{y}$.

${ }^{11}$ With normalized emittances defined as $\varepsilon_{n} \equiv \gamma_{o} \beta_{o} \varepsilon$.
}

$$
\begin{aligned}
& \left\langle\Delta \tilde{h}_{\mathrm{sc}}\right\rangle_{\varphi_{x, y}} \\
& =\frac{1}{\beta_{o}^{2} \gamma_{o}^{3}} \frac{Z^{2} N_{o} r_{c}}{\sqrt{2 \pi} \sigma_{z}} \mathrm{e}^{-\frac{\left(s-\beta_{o} \tau\right)^{2}}{2 \sigma_{z}^{2}}} \\
& \quad \cdot \int_{0}^{\infty} d \vartheta \frac{\left\langle\exp \left(-\frac{2 \beta_{x} I_{x} \cos ^{2} \varphi_{x}}{2(1+\vartheta / u) \sigma_{x}^{2}}\right)\right\rangle_{\varphi_{x}}\left\langle\operatorname { e x p } \left(-\frac{\left.\left.2 \beta_{y} I_{y} \cos ^{2} \varphi_{y}\right)\right\rangle_{\varphi_{y}}(1+\vartheta / u) \sigma_{y}^{2}}{\sqrt{(1+\vartheta / u)(1+\vartheta u)}}\right.\right.}{\sqrt{1+\vartheta}}
\end{aligned}
$$

and

$$
\begin{aligned}
\left\langle\Delta h_{\mathrm{sc}}\right\rangle_{\phi_{x}, \phi_{y}}= & \frac{1}{\beta_{o}^{2} \gamma_{o}^{3}} \frac{Z^{2} N_{o} r_{p}}{A \sqrt{2 \pi} \sigma_{z}} \mathrm{e}^{-\frac{\left(s-\beta_{o} \tau\right)^{2}}{2 \sigma_{z}^{2}}} \\
& \times \int_{0}^{\infty} d \vartheta \frac{F\left(\frac{A_{x}^{2} \cdot f_{E}}{2(1+\vartheta / u)}\right) F\left(\frac{A_{y}^{2}}{2(1+\vartheta u)}\right)}{\sqrt{(1+\vartheta / u)(1+\vartheta u)}},
\end{aligned}
$$

where we introduced new parameters:

$A_{x . y}^{2} \equiv \frac{I_{x, y}}{\varepsilon_{x, y}} ; \quad f_{E}(s)=\frac{\beta_{x}(s) \varepsilon_{x}}{\beta_{x}(s) \varepsilon_{x}+D_{x}^{2}(s) \sigma_{\delta}^{2}} \equiv \frac{\beta_{x}(s) I_{x}}{\sigma_{x}^{2} A_{x}^{2}}$.

As seen from Eq. (A18), the tune shifts depend on the horizontal and vertical actions as well as on the longitudinal position $s-\beta_{o} \tau$ inside the bunch. Averaging Eq. (A13) over the betatron phases gives the Hamiltonian of the linear motion:

$$
\begin{aligned}
\Delta \tilde{h}_{\mathrm{sc}}= & -\frac{1}{\beta_{o}^{2} \gamma_{o}^{3}} \frac{Z^{2} N_{o} r_{p}}{A \sqrt{2 \pi} \sigma_{z}} \mathrm{e}^{-\frac{\left(s-\beta_{o} \tau\right)^{2}}{2 \sigma_{z}^{2}}} \\
& \times\left(\frac{2 \beta_{x}(s) I_{x}\left\langle\cos ^{2}\left[\psi_{x}(s)+\phi_{x}\right]\right\rangle}{\sigma_{x}\left(\sigma_{x}+\sigma_{y}\right)}+\frac{\left\langle y^{2}\right\rangle}{\sigma_{y}\left(\sigma_{x}+\sigma_{y}\right)}\right)
\end{aligned}
$$

and

$$
\begin{aligned}
\left\langle\Delta h_{\mathrm{sc} L}\right\rangle= & -\frac{1}{\beta_{o}^{2} \gamma_{o}^{3}} \frac{Z^{2} N_{o} r_{p}}{A \sqrt{2 \pi} \sigma_{z}} \mathrm{e}^{-\frac{\left(s-\beta_{o} \tau\right)^{2}}{2 \sigma_{z}}} \frac{1}{\sigma_{x}(s)+\sigma_{y}(s)} \\
& \times\left(\frac{\beta_{x}(s) I_{x}}{\sigma_{x}(s)}+\frac{\beta_{y}(s) I_{y}}{\sigma_{y}(s)}\right) .
\end{aligned}
$$

We define the average of a periodic function ${ }^{12} g(s)=$ $g(s+C)$ as follows:

\footnotetext{
${ }^{12}$ We note that the lattice functions $\beta_{x, y}(s+C)=\beta_{x, y}(s)$ are periodic functions of the ring circumference, $\psi_{x, y}(s+C)=$ $\psi_{x, y}(s)+2 \pi Q_{x, y}$, while the betatron phases are monotonically growing functions.
} 


$$
\langle g\rangle_{C} \equiv \frac{1}{C} \int_{o}^{C} g(s) d s
$$

Using (A15) and (A20) we obtain the tune shifts experienced by particles near the axis that then are given by

$$
\begin{aligned}
\delta Q_{x} & =\frac{C}{2 \pi}\left\langle\frac{\partial}{\partial I_{x}}\left\langle\Delta h_{\mathrm{sc}}\right\rangle\right\rangle_{C} \\
& =-\frac{C}{2 \pi} \frac{1}{\beta_{o}^{2} \gamma_{o}^{3}} \frac{Z^{2} N_{o} r_{p}}{A \sqrt{2 \pi} \sigma_{z}} \mathrm{e}^{-\frac{\left(s-\beta_{o} \tau\right)^{2}}{2 \sigma_{z}^{2}}}\left\langle\frac{1}{\sigma_{x}(s)+\sigma_{y}(s)} \frac{\beta_{x}(s)}{\sigma_{x}(s)}\right\rangle_{C} \\
& =-\frac{C}{4 \pi} \frac{1}{\varepsilon_{x} \beta_{o}^{2} \gamma_{o}^{3}} \frac{Z^{2} N_{o} r_{p}}{A \sqrt{2 \pi} \sigma_{z}} \mathrm{e}^{-\frac{\left(s-\beta_{o} \tau\right)^{2}}{2 \sigma_{z}^{2}}}\left\langle\frac{2 f_{E}(s)}{1+\frac{1}{u}}\right\rangle_{C}
\end{aligned}
$$

to get

$$
\begin{aligned}
\delta Q_{x} & =-Q_{\mathrm{sc}}^{x} \cdot \mathrm{e}^{-\frac{\left(s-\beta_{o}\right)^{2}}{2 \sigma_{z}^{2}}}\left\langle\frac{2 \beta_{x}(s) \varepsilon_{x}}{\sigma_{x}(s)\left[\sigma_{x}(s)+\sigma_{y}(s)\right]}\right\rangle_{C} \\
& =-Q_{\mathrm{sc}}^{x} \cdot \mathrm{e}^{-\frac{\left(s-\beta_{o} \tau\right)^{2}}{2 \sigma_{z}^{2}}}\left\langle\frac{2 f_{E}(s) u(s)}{1+u(s)}\right\rangle_{C}, \\
\delta Q_{y} & =-Q_{\mathrm{sc}}^{y} \cdot \mathrm{e}^{-\frac{\left(s-\beta_{o} \tau\right)^{2}}{2 \sigma_{z}^{2}}}\left\langle\frac{2 \beta_{y}(s) \varepsilon_{y}}{\sigma_{y}(s)\left[\sigma_{x}(s)+\sigma_{y}(s)\right]}\right\rangle_{C} \\
& =-Q_{\mathrm{sc}}^{y} \cdot \mathrm{e}^{-\frac{\left(s-\beta_{o} \tau\right)^{2}}{2 \sigma_{z}^{2}}}\left\langle\frac{2}{1+u(s)}\right\rangle_{C},
\end{aligned}
$$

where we introduce approximate values for space-chargeinduced tune spreads as

$$
Q_{\mathrm{sc}}^{x, y}=\frac{C}{4 \pi \beta_{o}^{2} \gamma_{o}^{3} \varepsilon_{x, y}} \frac{Z^{2} N_{o} r_{p}}{A \sqrt{2 \pi} \sigma_{z}} .
$$

Similarly, using (A15), (A18), and (A19) we obtain the tune shifts for arbitrary amplitudes of betatron oscillations:

$$
\begin{aligned}
& \Delta Q_{\mathrm{sc} x}=Q_{\mathrm{sc}}^{x} \mathrm{e}^{-\frac{\left(s-\beta_{0} \tau\right)^{2}}{2 \sigma_{z}^{2}}} \cdot \int_{0}^{\infty} d \vartheta\left\langle f_{E} \frac{F^{\prime}\left(\frac{A_{x}^{2} \cdot f_{E}}{2(1+\vartheta / u)}\right) F\left(\frac{A_{y}^{2}}{2(1+\vartheta u)}\right)}{\sqrt{(1+\vartheta / u)^{3}(1+\vartheta u)}}\right\rangle_{C} \\
& \Delta Q_{\mathrm{sc} y}=Q_{\mathrm{sc}}^{y} \mathrm{e}^{-\frac{\left(s-\beta_{0} \tau\right)^{2}}{2 \sigma_{z}^{2}}} \cdot \int_{0}^{\infty} d \vartheta\left\langle\frac{F\left(\frac{A_{x}^{2} \cdot f_{E}}{2(1+\vartheta / u)}\right) F^{\prime}\left(\frac{A_{y}^{2}}{2(1+\vartheta u)}\right)}{\sqrt{(1+\vartheta / u)(1+\vartheta u)^{3}}}\right\rangle_{C}
\end{aligned}
$$

with $F^{\prime}(\xi)=\frac{d F(\xi)}{d \xi}=e^{-\xi}\left[I_{1}(\xi)-I_{o}(\xi)\right]$. The plot in Fig. 7 shows $F\left(A^{2}\right)$ and $-F^{\prime}\left(A^{2}\right)$ as functions of $A$. The behavior of the kernel in integral (A24), $F^{\prime} F$, indicates that the tune shift diminishes for particles with large oscillating amplitudes, e.g., $\Delta Q_{\text {sc } x} \rightarrow 0$ when $I_{x, y} \rightarrow \infty$. It also indicates

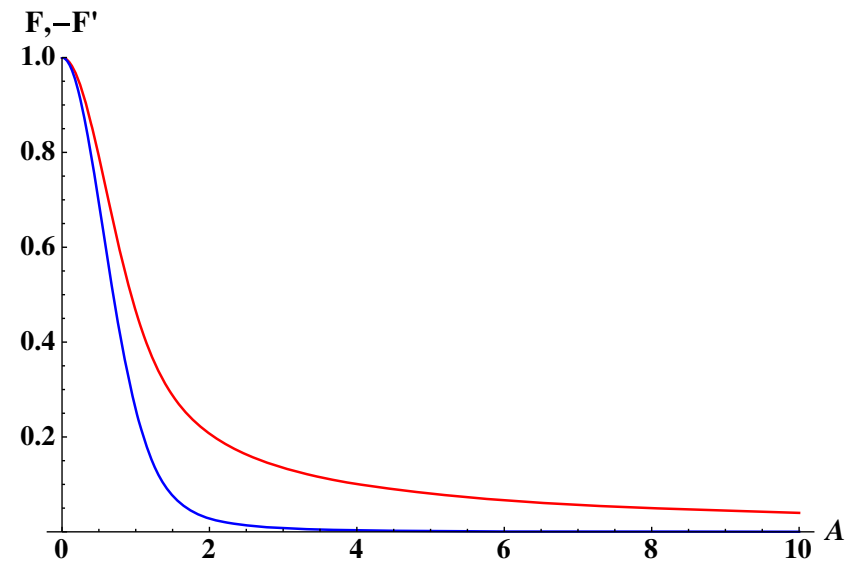

FIG. 7. Plots of $F\left(A^{2}\right)$ (red) and $-F^{\prime}\left(A^{2}\right)$ (blue) as a function of $A$ (horizontal axis).

that the tune spread for particles confined within $A_{x, y} \leq 5$ is close to that of the maximum shown in Eq. (A22).

For our further use in this paper we rewrite the expressions for the tune shifts as follows:

$$
\begin{aligned}
\Delta Q_{\mathrm{sc} x, y} & =-Q_{\mathrm{sc}}^{x, y} \cdot \mathrm{e}^{-\frac{\left(s-\beta_{0} \tau\right)^{2}}{2 \sigma_{z}^{2}}} \cdot f f_{x \cdot y}\left(\frac{I_{x}}{\varepsilon_{x}}, \frac{I_{y}}{\varepsilon_{y}}\right) ; \\
f f_{x}\left(A_{x}^{2}, A_{y}^{2}\right) & =-\int_{0}^{\infty} d \vartheta\left\langle f_{E} \frac{F^{\prime}\left(\frac{A_{x}^{2} \cdot f_{E}}{2(1+\vartheta / u)}\right) F\left(\frac{A_{y}^{2}}{2(1+\vartheta u)}\right)}{\sqrt{(1+\vartheta / u)^{3}(1+\vartheta u)}}\right\rangle_{C} ; \\
f f_{y}\left(A_{x}^{2}, A_{y}^{2}\right) & =-\int_{0}^{\infty} d \vartheta\left\langle\frac{F\left(\frac{A_{x}^{2} \cdot f_{E}}{2(1+\vartheta / u)}\right) F^{\prime}\left(\frac{A_{y}^{2}}{2(1+\vartheta u)}\right)}{\sqrt{(1+\vartheta / u)(1+\vartheta u)^{3}}}\right\rangle_{C}
\end{aligned}
$$

We note that both $f f_{x}$ and $f f_{y}$, while being smooth functions of $I_{x, y}$, are neither a simple exponential nor can they be expressed as well-known functions. This signifies that, in practice, they should be calculated (tabulated) for a specific storage ring.

Finally, we note that if the contribution of the energy spread term in (A17) is negligible, i.e.,

$$
\beta_{x, y}(s) \varepsilon_{x, y} \gg D_{x, y}^{2}(s) \sigma_{\delta}^{2},
$$

then both (A24) and (A25) can be reduced to "symmetrically looking" expressions with $f_{E}=1$. Further, for a round beam with equal transverse emittances, $\varepsilon_{x}=\varepsilon_{y}=\varepsilon$, we can write Eq. (A22) as follows:

$$
\delta Q_{x}=-Q_{\mathrm{sc}} \mathrm{e}^{-\frac{\left(s-\beta_{o}\right)^{2}}{2 \sigma_{z}^{2}}}\left\langle 2\left(1+\sqrt{\frac{\beta_{y}(s)}{\beta_{x}(s)}}\right)^{-1}\right\rangle_{C}
$$




$$
\delta Q_{y}=-Q_{\mathrm{sc}} \mathrm{e}^{-\frac{\left(s-\beta_{o} \tau\right)^{2}}{2 \sigma_{z}^{2}}}\left\langle 2\left(1+\sqrt{\frac{\beta_{x}(s)}{\beta_{y}(s)}}\right)^{-1}\right\rangle_{C},
$$

with the expressions

$$
Q_{\mathrm{sc}}=\frac{C}{4 \pi \beta_{o}^{2} \gamma_{o}^{3} \varepsilon} \frac{Z^{2} N_{o} r_{p}}{A \sqrt{2 \pi} \sigma_{z}},
$$

frequently used as an estimate for tune spread induced by space charge in hadron storage rings.

To connect the particle's distribution with the beam current, we should note that

$$
\begin{aligned}
I(s, \tau)= & \hat{I} \exp \left(-\frac{\left(s-\beta_{o} \tau\right)^{2}}{2 \sigma_{z}^{2}}\right) \\
\equiv & \hat{I} \exp \left(-\frac{\left(s / \mathrm{v}_{o}-t\right)^{2}}{2 \sigma_{t}^{2}}\right) \\
\hat{I} \equiv & \frac{e Z N_{o} \mathrm{v}_{o}}{(2 \pi)^{1 / 2} \sigma_{z}} ; \quad \sigma_{t}=\frac{\sigma_{z}}{\mathrm{v}_{o}} \\
f(x, y, s, \tau=c t)= & \frac{\hat{I}}{2 \pi \sigma_{x} \sigma_{y} Z e \mathrm{v}_{o}} \\
& \times \exp \left(-\frac{x^{2}}{2 \sigma_{x}^{2}}-\frac{y^{2}}{2 \sigma_{y}^{2}}-\frac{\left(s-\beta_{o} \tau\right)^{2}}{2 \sigma_{z}^{2}}\right),
\end{aligned}
$$

where $\hat{I}$ is the beam's peak current. This allows us to rewrite Eq. (A23) as

$$
\begin{aligned}
Q_{\mathrm{sc}}^{x, y} & =\frac{C}{4 \pi \beta_{o}^{3} \gamma_{o}^{3} \varepsilon_{x, y}} \frac{Z}{A} \frac{\hat{I}}{I_{p A}} ; \\
I_{p A} & =\frac{m_{p} c^{3}}{e} \equiv \frac{e c}{r_{p}} \cong 31.3 \times 10^{6} \mathrm{~A},
\end{aligned}
$$

where $I_{p A}$ is the Alfven current redefined for protons.

\section{APPENDIX B: TRANSVERSE DISTRIBUTION OF THE ELECTRON BEAM}

We are proposing to use a low energy electron beam to compensate the space- charge-induced tune shift and spread in a hadron beam with much higher energy. Both beams will have similar beam sizes and peak currents. Hence, the focusing effect of the hadron beam on the electron beam will be much stronger and, most likely, will cause pinching of the electron beam. The result of such an interaction will be neither desirable nor controllable. There is a practical way of keeping electron beam transverse distribution controllable and fixed [26] - the propagation of a magnetized beam in a strong solenoid field [78,79]. In this case, with a solenoid of an appropriately chosen strength, the electron beam's profile can be both maintained and controlled.

Similar to the arguments used for calculating the EM field induced by the hadron beam, we can conclude that the 4-potential induced by the electron beam is given by (A5) with a change of the kinematic variables $\gamma_{o} \rightarrow \gamma_{e} ; \beta_{o} \rightarrow \beta_{e}$ :

$$
\begin{aligned}
& {\left[\varphi_{e}(x, y, z, \tau), \vec{A}_{e}(x, y, z, \tau)\right]_{\mathrm{lab}}} \\
& \quad=\gamma_{e}\left(1, \beta_{e} \hat{s}\right) \cdot \bar{\varphi}_{e}\left[x, y, \gamma_{e}\left(z-\beta_{e} \tau\right)\right]
\end{aligned}
$$

with the scalar potential, $\bar{\varphi}_{e}$, satisfying the reduced Poison equation in the e-beam's comoving frame:

$$
\Delta_{\perp} \bar{\varphi}_{e}\left(\vec{r}_{\perp}, \bar{z}\right)=-4 \pi \rho_{e}\left(\vec{r}_{\perp}, \bar{z}\right) .
$$

As we discussed in the paper, the convolution of the longitudinal profile of the electron bunch has to fit that of the hadron bunch, while the transverse profile should be the same, i.e.,

$$
\begin{aligned}
\rho_{e}\left(\vec{r}_{\perp}, \bar{z}\right) & =\rho_{e \perp}\left(\vec{r}_{\perp}\right) \cdot \bar{g}(\bar{z}) ; \\
\bar{\varphi}_{e}\left(\vec{r}_{\perp}, \bar{z}\right) & =\bar{\varphi}_{e \perp}\left(\vec{r}_{\perp}\right) \cdot \bar{g}(\bar{z}) ; \\
\gamma_{e} \bar{g}(\bar{z}) & =g\left(z-\beta_{e} \tau\right) ; \\
\Delta_{\perp} \bar{\varphi}_{e \perp}\left(\vec{r}_{\perp}\right) & =-4 \pi \rho_{e \perp}\left(\vec{r}_{\perp}\right) .
\end{aligned}
$$

To satisfy the requirement for a desirable $\bar{\varphi}_{e}\left(\vec{r}_{\perp}\right)$, one "simply" must provide a beam with transverse density distribution satisfying

$$
\rho_{e \perp}\left(\vec{r}_{\perp}\right)=-\frac{\Delta_{\perp} \bar{\varphi}_{e \perp}\left(\vec{r}_{\perp}\right)}{4 \pi} .
$$

It is easy to see that negatively charged beam is needed to compensate for the space charge of positively charged particles, e.g., we know the sign of $\rho_{e}<0$. But while looking as a simple mathematical relationship (i.e., the double differentiation of a given function), we must take into account that the density should not change the sign, i.e., on potentials with (unless the ignored longitudinal part of the Poisson equation becomes non-negligible for some reason)

$$
\Delta_{\perp} \bar{\varphi}_{e}\left(\vec{r}_{\perp}\right) \geq 0 ; \quad \forall \vec{r}_{\perp}
$$

are allowed in practice. Unfortunately, this is not the only limitation; generating an arbitrary profile of the electron bunch is a nontrivial engineering undertaking.

While there are practical challenges in generating the desirable transverse profiles (especially when the current is modulated), a large diversity of transverse profiles already has been generated in practice $[64,78,79]$. Hence, we can assume a smooth profile mimicking to a significant degree the distribution of a hadron beam can be produced. 
This most likely means that only the main features of the space-charge-induced tune spread can be mimicked by the compensating beam, and only partial compensation of the tune spread would be possible. The degree of compensation will depend both on the ring, and the attainable profile of the electron beam.

\section{APPENDIX C: TUNE SHIFT INDUCED IN THE E-BEAM COMPENSATOR}

Next, we consider an electron beam in a compensating scheme with a known 4-potential [see (B1)]:

$$
\begin{aligned}
& {\left[\varphi_{e}(x, y, s, \tau), \vec{A}_{e}(x, y, s, \tau)\right]_{\mathrm{lab}}} \\
& \quad=\gamma_{e}\left(1, \beta_{e} \hat{s}\right) \cdot \bar{\varphi}_{e}\left[x, y, \gamma_{e}\left(s-\beta_{e} \tau\right)\right]
\end{aligned}
$$

and according to (B2) we get

$$
\begin{aligned}
& {\left[\varphi_{e}(x, y, s, \tau), \vec{A}_{e}(x, y, s, \tau)\right]_{\mathrm{lab}}} \\
& \quad=\gamma_{e}\left(1, \beta_{e} \hat{s}\right) \cdot \bar{\varphi}_{e \perp}(x, y) \cdot g\left(s-\beta_{e} \tau\right) ; \\
& \quad \int_{-\infty}^{\infty} g(s) d s=1 .
\end{aligned}
$$

The latter can be input into the transverse accelerator Hamiltonian for the hadrons (similarly to the procedure in Appendix A) to find how it changes:

$$
\delta \tilde{h}_{e}=\frac{Z e \gamma_{e}}{\beta_{0} p_{o} c}\left(1-\beta_{e} \beta_{0}\right) \bar{\varphi}_{e \perp}(x, y) \cdot g\left(s-\beta_{e} \tau\right) .
$$

Assuming the symmetry of the electron beam distribution, we can assume that

$$
\bar{\varphi}_{e}(x, y)=\bar{\varphi}\left(x^{2}, y^{2}\right)
$$

and we can average the Hamiltonian over the betatron phases to find the effective one:

$$
\begin{aligned}
\left\langle\delta h_{e}\right\rangle= & \frac{Z \gamma_{e} e}{\beta_{0} p_{o} c}\left(1-\beta_{e} \beta_{0}\right) \\
& \times\left\langle\bar{\varphi}\left[2 I_{x} \beta_{x}(s) \cos ^{2} \phi_{x}, 2 I_{y} \beta_{y}(s) \cos ^{2} \phi_{y}\right]\right\rangle_{\phi_{x, y}} \\
& \cdot g\left(s-\beta_{e} \tau\right) .
\end{aligned}
$$

The effective Hamiltonian for a hadron traversing the interaction region can be expressed as the following:

$$
\begin{aligned}
s & =\beta_{o} \cdot\left(\tau-\tau_{o}\right) ; \quad \tau=\tau_{o}+\frac{s}{\beta_{o}} \\
\int_{-L / 2}^{L / 2} d s\left\langle\delta h_{e}\right\rangle & =\frac{Z e \gamma_{e}}{\beta_{0}^{2} \gamma_{o} A m c^{2}}\left(1-\beta_{e} \beta_{0}\right) \int_{-L / 2}^{L / 2} d s\left\langle\bar{\varphi}\left[2 I_{x} \beta_{x}(s) \cos ^{2} \phi_{x}, 2 I_{y} \beta_{y}(s) \cos ^{2} \phi_{y}\right]\right\rangle_{\phi_{x, y}} \cdot g\left[s\left(1-\frac{\beta_{e}}{\beta_{0}}\right)-\beta_{e} \tau_{o}\right] .
\end{aligned}
$$

Further evaluation is impossible without knowing the specific transverse distribution of electron beam. It is natural to assume that it also is Gaussian,

$$
\begin{array}{r}
f_{e}(x, y, s, \tau=c t)=\frac{N_{e}}{(2 \pi)^{3 / 2} \sigma_{x} \sigma_{y}} \exp \left(-\frac{x^{2}}{2 \sigma_{x}^{2}}-\frac{y^{2}}{2 \sigma_{y}^{2}}\right) g\left(s-\beta_{e} \tau\right) ; \\
\varphi(\vec{r})=-e N_{e} g\left(s-\beta_{e} \tau\right) \int_{0}^{\infty} \frac{e^{-\frac{x^{2}}{2 \sigma_{e x}^{2}+q}-\frac{y^{2}}{2 \sigma_{e y}^{2}+q}}}{\sqrt{\left(2 \sigma_{e x}^{2}+q\right)\left(2 \sigma_{e y}^{2}+q\right)}} d q,
\end{array}
$$

and continue with the Hamiltonian variation of

$$
\begin{aligned}
\left\langle\Delta h_{e}\right\rangle & =\frac{Z N_{e} r_{p}}{\beta_{0}^{2} \gamma_{o} A}\left(1-\beta_{e} \beta_{0}\right) \cdot g\left[s\left(1-\frac{\beta_{e}}{\beta_{0}}\right)-\beta_{e} \tau_{o}\right] \times \int_{0}^{\infty} d q \frac{F\left(\frac{\beta_{x}(s) I_{x}}{q+2 \sigma_{x}^{2}}\right) F\left(\frac{\beta_{y}(s) I_{y}}{q+2 \sigma_{y}^{2}}\right)}{\sqrt{\left(q+2 \sigma_{x}^{2}\right)\left(q+2 \sigma_{y}^{2}\right)}} \\
F(x) & =I_{o}(x) e^{-x}
\end{aligned}
$$

and the induced integral Hamiltonian is 


$$
\Delta H=\int_{-L / 2}^{L / 2}\left\langle\Delta h_{e}\right\rangle d s=\frac{Z N_{e} r_{p}}{\beta_{0}^{2} \gamma_{o} A}\left(1-\beta_{e} \beta_{0}\right) \times \int_{-L / 2}^{L / 2} d s g\left[s\left(1-\frac{\beta_{e}}{\beta_{0}}\right)-\beta_{e} \tau_{o}\right] \int_{0}^{\infty} d q \frac{F\left(\frac{\beta_{x}(s) I_{x}}{q+2 \sigma_{x}^{2}}\right) F\left(\frac{\beta_{y}(s) I_{y}}{q+2 \sigma_{y}^{2}}\right)}{\sqrt{\left(q+2 \sigma_{x}^{2}\right)\left(q+2 \sigma_{y}^{2}\right)}}
$$

and the induced tune shifts are given by following convolutions:

$$
\begin{gathered}
\Delta Q_{x}=\frac{Z N_{e} r_{p}}{2 \pi \beta_{0}^{2} \gamma_{o} A}\left(1-\beta_{e} \beta_{0}\right) \int_{-L / 2}^{L / 2} d s g(\xi) \int_{0}^{\infty} d q \beta_{x} \frac{F^{\prime}\left(\frac{\beta_{x} I_{x}}{q+2 \sigma_{x}^{2}}\right) F\left(\frac{\beta_{y} I_{y}}{q+2 \sigma_{y}^{2}}\right)}{\sqrt{\left(q+2 \sigma_{x}^{2}\right)^{3}\left(q+2 \sigma_{y}^{2}\right)}} ; \\
\Delta Q_{x}=\frac{Z N_{e} r_{p}}{2 \pi \beta_{0}^{2} \gamma_{o} A}\left(1-\beta_{e} \beta_{0}\right) \int_{-L / 2}^{L / 2} d s g(\xi) \int_{0}^{\infty} d q \beta_{y} \frac{F\left(\frac{\beta_{x} I_{x}}{q+2 \sigma_{x}^{2}}\right) F^{\prime}\left(\frac{\beta_{y} I_{y}}{q+2 \sigma_{y}^{2}}\right)}{\sqrt{\left(q+2 \sigma_{x}^{2}\right)\left(q+2 \sigma_{y}^{2}\right)^{3}}} ; \\
\xi=s\left(1-\frac{\beta_{e}}{\beta_{0}}\right)-\beta_{e} \tau_{o} .
\end{gathered}
$$

One possible simplification to (C9) can be done for a case when the size of an electron beam stays constant through the interaction region, and $\beta_{x, y} \gg L$, that is, we can use a thin-lens approximation for the kick. In this approximation, the effect of the slippage and that of the kick are separable:

$$
\begin{aligned}
\Delta Q_{x} \cong \frac{Z N_{e} r_{p} \bar{\beta}_{x}}{2 \pi \beta_{0}^{2} \gamma_{o} A}\left(1-\beta_{e} \beta_{0}\right) G(\tau) \int_{0}^{\infty} d q \frac{F^{\prime}\left(\frac{\bar{\beta}_{x} I_{x}}{q+2 \sigma_{x}^{2}}\right) F\left(\frac{\bar{\beta}_{y} I_{y}}{q+2 \sigma_{y}^{2}}\right)}{\sqrt{\left(q+2 \sigma_{x}^{2}\right)^{3}\left(q+2 \sigma_{y}^{2}\right)}} \\
\Delta Q_{y} \cong \frac{Z N_{e} r_{p} \bar{\beta}_{y}}{2 \pi \beta_{0}^{2} \gamma_{o} A}\left(1-\beta_{e} \beta_{0}\right) G(\tau) \int_{0}^{\infty} d q \frac{F\left(\frac{\bar{\beta}_{x} I_{x}}{q+2 \sigma_{x}^{2}}\right) F^{\prime}\left(\frac{\bar{\beta}_{y} I_{y}}{q+2 \sigma_{y}^{2}}\right)}{\sqrt{\left(q+2 \sigma_{x}^{2}\right)\left(q+2 \sigma_{y}^{2}\right)^{3}}} ; \\
G(\tau)=\int_{-L / 2}^{L / 2} d s g\left[\left(1-\frac{\beta_{e}}{\beta_{0}}\right) s-\beta_{e} \tau\right] ; \quad \bar{\beta}_{x, y}=\frac{1}{L} \int_{-L / 2}^{L / 2} \beta_{x, y}(s) d s .
\end{aligned}
$$

The idea of the method is that $G(\tau)$ approximates the hadron beam's longitudinal shape. Then the goal of the transverse shaping and choosing an appropriate $\beta_{x, y}$ as well as the electron beam intensity and sizes is to approximate the values of the tune shift induced by the space-charge effects and their dependence on the transverse actions. These assumptions set already familiar requirements:

$$
G(\tau)=e^{-\frac{\left(s-\beta_{o} \tau\right)^{2}}{2 \sigma_{z}^{2}}} ; \quad N_{e}=\frac{Z N_{o}}{\gamma_{o}^{2}\left(1-\beta_{e} \beta_{0}\right)} \frac{C}{\sqrt{2 \pi} \sigma_{z}} .
$$

\section{APPENDIX D: DECONVOLUTION}

Before starting the derivation for deconvolving Eq. (22), we list our assumptions:

1. By definition, $\Delta z=c \Delta t>0$.

2. We assume that the hadron beam longitudinal distribution function, $q(z)$, is an analytical function, with a finite integral and values diminishing at infinity.
3. Furthermore, we assume that at large distances, its derivative also vanishes faster that $1 /|z|$, that is $\left|q^{\prime}(z)\right|<A /|z|^{1+\varepsilon}, \varepsilon>0$. This rather weak assumption will be used for proving the convergence of the convolution.

To deconvolve the equation (22), we rewrite it in the following form:

$$
\begin{gathered}
\int_{0}^{\Delta z} g(z+\zeta) d \zeta=q(z) \\
\Downarrow \\
\int_{z}^{z+\Delta z} g(\zeta) d \zeta=q(z) .
\end{gathered}
$$

Taking the derivative, we get the finite step differential equation on $g$ :

$$
g(z+\Delta z)-g(z)=q^{\prime}(z)
$$

which can be solved by turning the finite series 


$$
\begin{aligned}
\sum_{m=0}^{n} q^{\prime}(z+m \Delta z) & =\sum_{m=0}^{n} g[z+(m+1) \Delta z]-\sum_{m=0}^{n} g(z+m \Delta z) \\
& =\sum_{m=1}^{n+1} g(z+m \Delta z)-\sum_{m=0}^{n} g(z+m \Delta z) \\
\sum_{m=0}^{n} q^{\prime}(z+m \Delta z) & =g[z+(n+1) \Delta z]-g(z)
\end{aligned}
$$

and assuming that $g(z)_{z \rightarrow \infty} \rightarrow 0$, we can derive the result:

$$
g_{+}(z)=-\sum_{m=0}^{\infty} q^{\prime}(z+m \Delta z) .
$$

We naturally assume that the sum $\sum_{m=0}^{\infty} q^{\prime}(z+m \Delta z)$ converges, i.e., that the derivative of the distribution function at large values falls faster than $A /|z|^{1+\varepsilon}, \varepsilon>0$. Similarly, using

$$
\begin{aligned}
\sum_{m=1}^{n+1} q^{\prime}(z-m \Delta z) & =\sum_{m=0}^{n} g(z-m \Delta z)-\sum_{m=1}^{n+1} g(z-m \Delta z) \\
& =g(z)-g[z-(n+1) \Delta z],
\end{aligned}
$$

and assuming that $g(z)_{z \rightarrow-\infty} \rightarrow 0$, we can derive the second result:

$$
g_{-}(z)=+\sum_{m=1}^{\infty} q^{\prime}(z-m \Delta z) .
$$

Since the function $g(z)$ is not unique, function $g_{-}$in (D6) is not necessarily identical to $g_{+}$in (D4). Adding any periodical function with period $\Delta z$ and a zero integral, we get

$$
\begin{aligned}
g_{1}(z) & =g(z)+p(z) \\
p(z+\Delta z) & =p(z) ; \quad \int_{0}^{\Delta z} p(z) d z=0
\end{aligned}
$$

does not change the property of satisfying the convolution. But it definitely violates the natural requirement that $g(z)_{z \rightarrow \pm \infty} \rightarrow 0$, i.e., the length of the electron bunch is finite.

By observing (D4) and (D6), we can conclude that for a properly behaving distribution function

$$
\left|q^{\prime}(z)\right|<A /|z|^{1+\varepsilon}, \quad \varepsilon>0
$$

$g_{+}(z)_{z \rightarrow+\infty} \rightarrow 0$ and $g_{-}(z)_{z \rightarrow-\infty} \rightarrow 0$. It is rather easy to prove by considering $z>0$ :

$$
\begin{aligned}
&\left|g_{+}(z>0)\right|<\sum_{m=0}^{\infty}\left|q^{\prime}(z+m \Delta z)\right|<A \sum_{m=0}^{\infty}|z+m \Delta z|^{-(1+\varepsilon)}<A \sum_{m=M}^{\infty}|m \Delta z|^{-(1+\varepsilon)}=\frac{A}{\Delta z^{1+\varepsilon}} \sum_{m=M}^{\infty} \frac{1}{m^{1+\varepsilon}} ; \\
& M=\text { Floor }\left[\frac{z}{\Delta z}\right]>\frac{z-\Delta z}{\Delta z} ; \\
& \sum_{m=M}^{2 M-1} \frac{1}{m^{1+\varepsilon}}<\sum_{m=M}^{2 M-1} \frac{1}{M^{1+\varepsilon}}=\frac{1}{M^{\varepsilon}} ; \quad \sum_{m=2 M}^{4 M-1} \frac{1}{(2 M)^{1+\varepsilon}}<\frac{1}{(2 M)^{\varepsilon}} \rightarrow \sum_{m=M}^{\infty} \frac{1}{m^{1+\varepsilon}} \leq \frac{1}{M^{\varepsilon}} \sum_{n=0}^{\infty} \frac{1}{\left(2^{\varepsilon}\right)^{n}}=\frac{1}{M^{\varepsilon}} \frac{2^{\varepsilon}}{2^{\varepsilon}-1} ; \\
&\left|g_{+}(z>0)\right|<\frac{A}{\Delta z^{1+\varepsilon}} \frac{1}{M^{\varepsilon}} \frac{2^{\varepsilon}}{2^{\varepsilon}-1}<\frac{A}{\Delta z^{1}} \frac{1}{(z-\Delta z)^{\varepsilon}} \frac{2^{\varepsilon}}{2^{\varepsilon}-1}
\end{aligned}
$$

with the latter expression definitely showing that $g_{+}(z)_{z \rightarrow+\infty} \rightarrow 0$. Proving $g_{-}(z)_{z \rightarrow-\infty} \rightarrow 0$ is identical to the logic with that given above, but for $z<0$ replacing $M$ to $M=$ Floor $\left[-\frac{z}{\Delta z}\right]>-\frac{z+\Delta z}{\Delta z}$.

While at some values of $\Delta z g_{-}$can be identical to $g_{+}$, there are clear physics examples of where they can diverge. For example, we can consider a bell-shaped $q(z)$ with its entire span falling within $\Delta z$, i.e., $q( \pm \Delta z / 2)=0$. In practice such distributions exist, for example as a bunch in a single rf bucket. It means that 
At the same time $q^{\prime}(z)$ has at least one maximum value and one minimum one, with an interval $\{-\Delta z / 2, \Delta z / 2\}$. We mark the locations of these $q^{\prime}(z)$ extremes as $z_{+}\left(\right.$maximum,$\left.z_{+}<0\right), z_{-}\left(\right.$minimum, $\left.z_{-}>0\right)$. Then, for an arbitrarily positive $m$, we have $g_{+}(z)=$ $-\sum_{m=0}^{\infty} q^{\prime}(z+m \Delta z), g_{-}(z)=+\sum_{m=1}^{\infty} q^{\prime}(z-m \Delta z)$ :

$$
\begin{aligned}
& g_{+}\left(z_{+}-m \Delta z\right)=-q^{\prime}\left(z_{+}\right)<0 ; \\
& g_{+}\left(z_{-}-m \Delta z\right)=-q^{\prime}\left(z_{-}\right)>0 ; \\
& g_{-}\left(z_{+}+m \Delta z\right)=q^{\prime}\left(z_{+}\right)>0 ; \\
& g_{-}\left(z_{-}+m \Delta z\right)=q^{\prime}\left(z_{-}\right)<0,
\end{aligned}
$$

i.e., denoting the oscillating nature of $g_{+}$at negative values and $g_{-}$at positive values of the argument. Furthermore,

$$
\begin{aligned}
& g_{+}(z)=0 ; \quad z>\Delta z / 2, \\
& g_{+}(z)=-q^{\prime}[\Delta z \bmod (z / \Delta z+1 / 2)-\Delta z / 2], \quad z<\Delta z / 2, \\
& g_{-}(z)=0 ; \quad z<\Delta z / 2, \\
& g_{-}(z)=q^{\prime}[\Delta z \bmod (z / \Delta z+1 / 2)-\Delta z / 2], \quad z>\Delta z / 2, \\
& g_{+}(z)-g_{-}(z)=-q^{\prime}[\Delta z \bmod (z / \Delta z+1 / 2)-\Delta z / 2],
\end{aligned}
$$

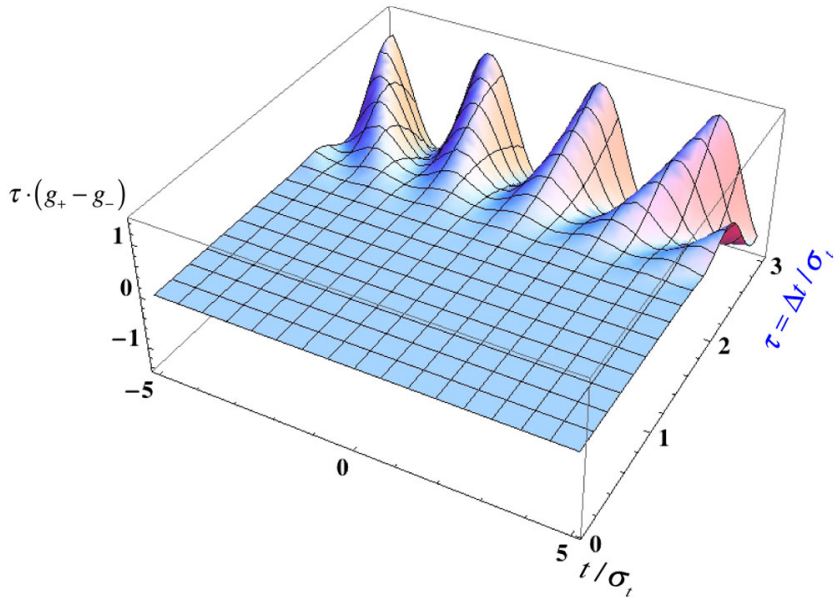

FIG. 8. 3D plot of $\tau \cdot\left[g_{+}(t)-g_{-}(t)\right]$ (vertical axis) for Gaussian convolution function with $t=z / \sigma_{z} \in(-5,5)$ being a horizontal axis, and the third axis is $\tau=\Delta z / \sigma_{z} \in\{0,3\}$ [see Eqs. (D4) and (D6)].

with the difference being a periodic function with zerovalue integral. Figure 8 below shows this behavior for a Gaussian $q(z)$ as a function of the delay.

Since Eq. (22) is linear, any combination of

$$
g_{\alpha}(t)=\alpha g_{+}(t)+(1-\alpha) g_{-}(t)
$$

is a deconvolution of Eq. (22). For practical applications, the most interesting is an even sum of both:

$$
\begin{aligned}
2 g_{1 / 2}(z) & =g_{+}(z)+g_{-}(z)=\sum_{m=1}^{\infty} q^{\prime}(z-m \Delta z)-\sum_{m=0}^{\infty} q^{\prime}(z+m \Delta z), \\
2 g_{1 / 2}\left(z+\frac{\Delta z}{2}\right) & =\sum_{m=1}^{\infty} q^{\prime}\left(z-m \Delta z+\frac{\Delta z}{2}\right)-\sum_{m=0}^{\infty} q^{\prime}\left(z+m \Delta z+\frac{\Delta z}{2}\right), \\
\sum_{m=0}^{\infty} q^{\prime}\left(z+m \Delta z+\frac{\Delta z}{2}\right) & =\sum_{m=1}^{\infty} q^{\prime}\left(z+m \Delta z-\frac{\Delta z}{2}\right) \\
2 g_{1 / 2}\left(z+\frac{\Delta z}{2}\right) & =\sum_{m=1}^{\infty}\left\{q^{\prime}\left[z-\left(m-\frac{1}{2}\right) \Delta z\right]-q^{\prime}\left[z+\left(m-\frac{1}{2}\right) \Delta z\right]\right\} .
\end{aligned}
$$

Hence, we can define a "symmetric-form" function as

$$
G(z)=g\left(z+\frac{\Delta z}{2}\right)=\frac{1}{2} \sum_{m=1}^{\infty}\left\{q^{\prime}\left[z-\left(m-\frac{1}{2}\right) \Delta z\right]-q^{\prime}\left[z+\left(m-\frac{1}{2}\right) \Delta z\right]\right\}
$$

and easily prove that 


$$
\begin{aligned}
& \sum_{m=1}^{\infty} \int_{z-\frac{\Delta z}{2}}^{z+\frac{\Delta z}{2}} d z_{1}\left\{q^{\prime}\left[z_{1}-\left(m-\frac{1}{2}\right) \Delta z\right]-q^{\prime}\left[z_{1}+\left(m-\frac{1}{2}\right) \Delta z\right]\right\} \\
& =\sum_{m=1}^{\infty}\{q(z+\Delta z-m \Delta z)-q(z+m \Delta z)-q(z-m \Delta z)+q(z-\Delta z+m \Delta z)\} \\
& =\left\{\sum_{m=0}^{\infty} q(z-m \Delta z)-\sum_{m=1}^{\infty} q(z+m \Delta z)-\sum_{m=1}^{\infty} q(z-m \Delta z)+\sum_{m=0}^{\infty} q(z+m \Delta z)\right\}=2 q(z)
\end{aligned}
$$

$$
\int_{z-\Delta z / 2}^{z+\Delta z / 2} G(\zeta) d \zeta=q(z)
$$

If $q(-z)=q(z)$ is symmetric, then

$$
\begin{aligned}
q^{\prime}(z) & =-q^{\prime}(-z) \rightarrow q^{\prime}\left[z-\left(m-\frac{1}{2}\right) \Delta z\right]=-q^{\prime}\left[\left(m-\frac{1}{2}\right) \Delta z-z\right], \\
q^{\prime}\left[z+\left(m-\frac{1}{2}\right) \Delta z\right] & \equiv q^{\prime}\left[\left(m-\frac{1}{2}\right) \Delta z+z\right]
\end{aligned}
$$

and $G(z)$ can be rewritten in a form that has obviously symmetric terms:

$$
G(z)=-\frac{1}{2} \sum_{m=1}^{\infty}\left\{q^{\prime}\left[\left(m-\frac{1}{2}\right) \Delta z-z\right]+q^{\prime}\left[\left(m-\frac{1}{2}\right) \Delta z+z\right]\right\} .
$$

It is easy to prove:

$$
\begin{aligned}
v(z) & =q^{\prime}(a-z)+q^{\prime}(a+z) ; \\
v(-z) & =q^{\prime}(a+z)+q^{\prime}(a-z) \equiv v(z) .
\end{aligned}
$$

Let us consider a practical case with

$$
q(z)=\exp \left(-\frac{z^{2}}{2 \sigma^{2}}\right)
$$

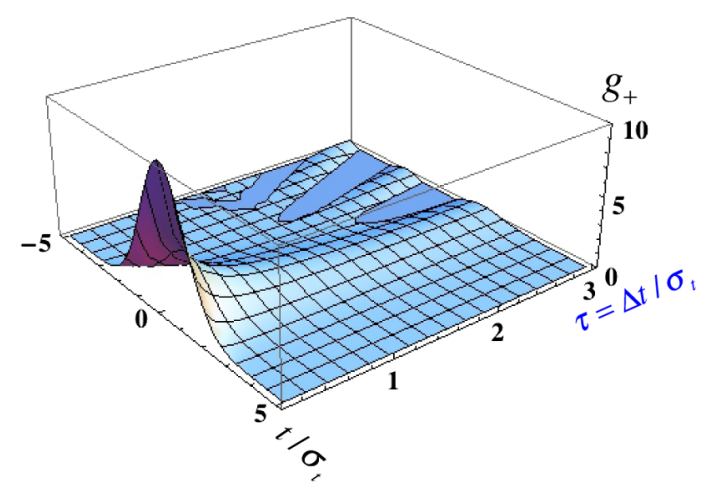

(a)

$$
\text { we then have }
$$

$$
g_{+}(z)=\frac{1}{\sigma^{2}} \sum_{n=0}^{\infty}(z+n \Delta z) \cdot \exp \left(-\frac{(z+n \Delta z)^{2}}{2 \sigma^{2}}\right)
$$

Undoubtedly, the behavior of the $g(z)$ is defined by dimensionless parameter $\tau=\frac{\Delta z}{\sigma}$ :

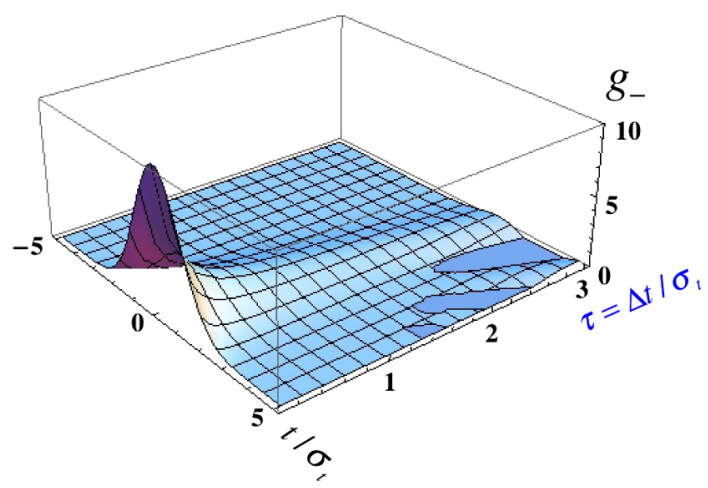

(b)

FIG. 9. 3D plots of (a) $g_{+}(t)$ and (b) $g_{-}(t)$ as functions of $t \in(-5,5)$ and the parameter $\tau \in\{0,3\}$. The clipping shows the area where the function becomes negative. 


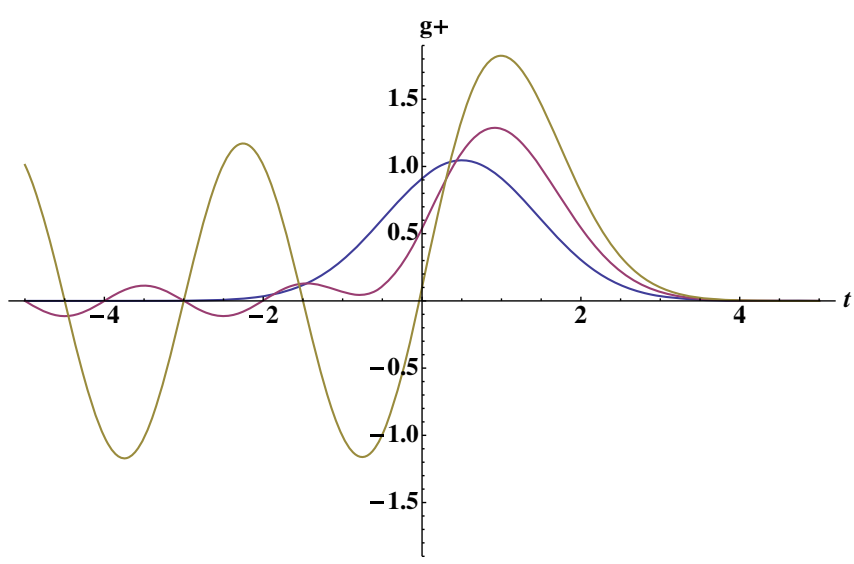

(a)

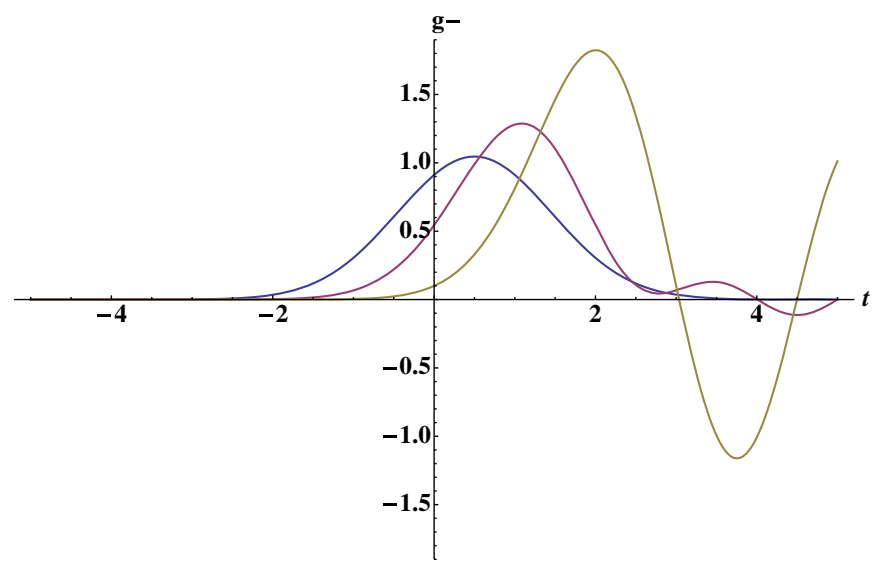

(b)

FIG. 10. Plot of $\tau \cdot g_{ \pm}(t)\left(g_{+}\right.$is on the left, and $g_{-}$is on the right) as functions of $t \in(-5,5)$. Colors code: blue for $\tau=1$, cyan for $\tau=2$ and yellow-green for $\tau=3$.

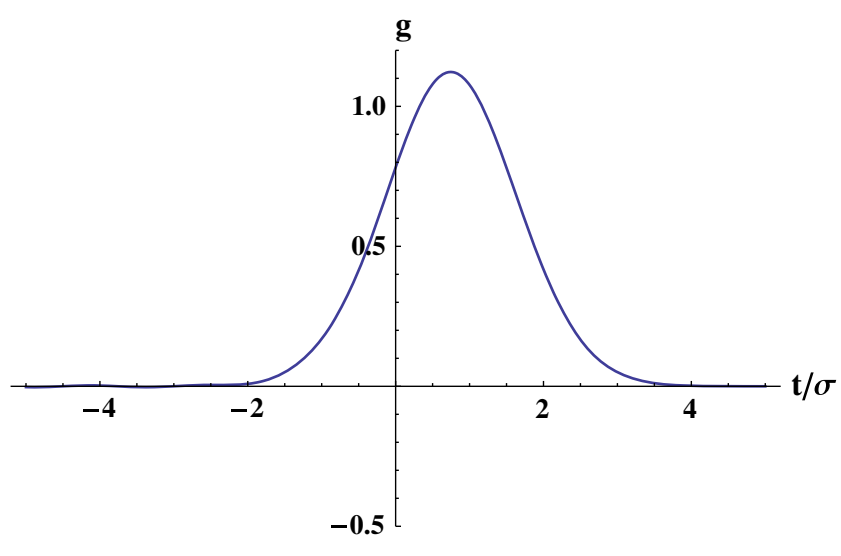

FIG. 11. 3D plot of $\tau g_{+}(t)$ as a function of $t \in(-5,5)$ and for $\tau=1.5$.

$$
\begin{aligned}
g_{+}(t) & =\frac{1}{\sigma} \sum_{n=0}^{\infty}(t+n \tau) \cdot \exp \left(-\frac{(t+n \tau)^{2}}{2}\right) ; \\
t & =\frac{z}{\sigma} ; \quad \tau=\frac{\Delta z}{\sigma} .
\end{aligned}
$$

Figure 9 shows the value of $g_{ \pm}(t)$ as functions of $t \in(-5,5)$ and $\tau \in\{0,3\}$. It shows that for $\tau \in\{0,1\}$ the functions $g_{ \pm}(t)$ remain positive. Figure 10 shows details of $g_{ \pm}(t)$ at $\tau=1,2,3$. Again, it is obvious that for $\tau>2$ it is impossible to make $g(t)>0$ approximating the required function. But it also is obvious that for $\tau \leq 1.5$ Equations (D4) and (D6) generate a smooth positive function, which, in principle, can be reproduced by an e-beam's profile.

Figures 3-5 in Sec. III also show that deconvolutions for $\tau \leq 1.5$ are generating smooth positive functions closely approximating the required forms. As shown in Fig. 11, the $g_{+}(t)$ for $\tau=1.5$ attains small negative values

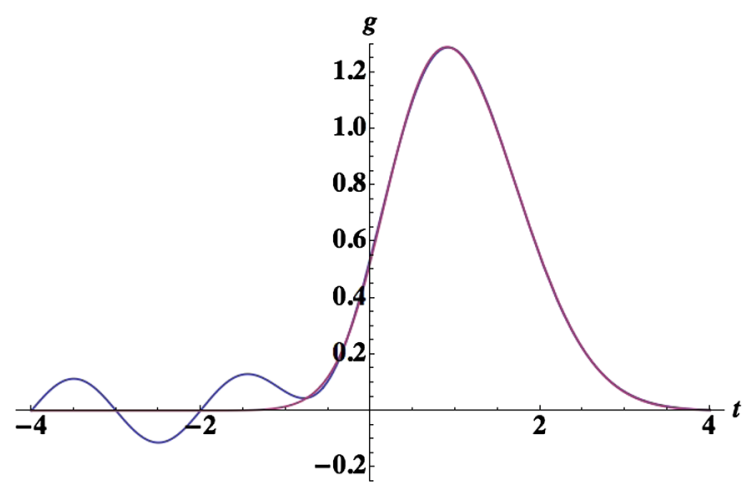

(a)

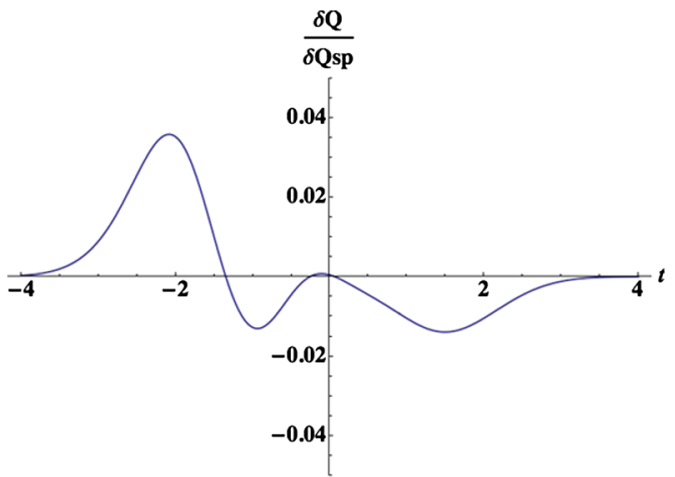

(b)

FIG. 12. (a) A plot of $g_{+}(t)$ for $\tau=2$ (blue) and a fit of a positive function (magenta). (b) The residual tune shift as a function of the position within a hadron bunch, when a fitted function is used. 
at $t<-3$. Detailed studies show that it becomes an oscillating sin-like function with amplitude about $1.5 \times 10^{-3}$ and with period of $T \sim 1.5$. Simply cutting this tail at $t<-3$ makes a practically useful positive function, whose convolution deviates from (22) only by a small fraction $\sim 7 \times 10^{-4}$.

As Fig. 12 shows, it even is possible to use $\tau=2$ with a smooth positive function, approximating the exact deconvolution, to compensate for more than $95 \%$ of the tune spread induced by the space charge. While this can be considered, using $\tau \leq 1.5$ is preferable for accurate spacecharge compensation.

[1] V.N. Litvinenko et al., Report No. BNL-96164-2011, 2011, pp. 436-443, http://arxiv.org/pdf/1108.1713v1.

[2] L. J. Laslett, BNL Report No. 7535, 1963, p. 154, http://lss .fnal.gov/conf/C630610/p324.pdf.

[3] C. E. Nielsen and A. M. Sessler, Rev. Sci. Instrum. 30, 80 (1959).

[4] S. Machida, Nucl. Instrum. Methods Phys. Res., Sect. A 309, 43 (1991).

[5] S. G. Anderson, J. B. Rosenzweig, G. P. LeSage, and J. K. Crane, Phys. Rev. ST Accel. Beams 5, 014201 (2002).

[6] A. V. Fedotov, in Proceedings of the 2003 Particle Accelerator Conference, Portland, OR (IEEE, New York, 2003), p. 383.

[7] B. W. Montague, CERN Report No. 68-38, 1968.

[8] I. Hofmann, Phys. Rev. E 57, 4713 (1998).

[9] S. Machida, Nucl. Instrum. Methods Phys. Res., Sect. A 384, 316 (1997).

[10] I. Hofmann and K. Beckert, in Proceedings of the 11th Particle Accelerator Conference, PAC-1985, Vancouver, BC, Canada, 1985 (IEEE, New York, 1985), p. 2264.

[11] F. Sacherer, LNBL Report No. UCRL-18454, 1968.

[12] R. L. Gluckstern, in Proceedings of 1970 Proton Linear Accelerator Conference, Batavia IL, 1970, p. 811.

[13] I. Hofmann et al., in Proceedings of the 8th European Particle Accelerator Conference, Paris, 2002 (EPS-IGA and CERN, Geneva, 2002), p. 74.

[14] A. V. Fedotov, J. Holmes, and R. L. Gluckstern, Phys. Rev. ST Accel. Beams 4, 084202 (2001).

[15] J.S. O'Connell et al., in Proceedings of Particle Accelerator Conference, Washington DC, 1993 (IEEE, Piscataway, NJ, 1993), p. 3657.

[16] J. Qiang and R. D. Ryne, Phys. Rev. ST Accel. Beams 3, 064201 (2000).

[17] J. M. Lagniel, Nucl. Instrum. Methods Phys. Res., Sect. A 345, 46 (1994); 345, 405 (1994).

[18] A. Riabko, M. Ellison, X. Kang, S. Lee, D. Li, J. Liu, X. Pei, and L. Wang, Phys. Rev. E 51, 3529 (1995).

[19] M. S. Livingston, MIT Report No. 60, 1953, p. 154.

[20] H. Okamoto and K. Yokoya, Nucl. Instrum. Methods Phys. Res., Sect. A 482, 51 (2002).

[21] I. Hofmann et al., Part. Accel. 13, 145 (1983).

[22] J. Struckmeier and M. Reiser, Part. Accel. 14, 227 (1984).
[23] Handbook of Accelerator Physics, and Engineering, edited byA. Chao and M. Tigner, 2nd ed. (World Scientific Publishing Co., Singapore, 2002), p. 128.

[24] A. V. Burov, G.W. Foster, and V.D. Shiltsev, Report No. FERMILAB-TM-2125, 2000.

[25] A. Shemyakin et al., in Proceedings of the European Particle Accelerator Conference, Vienna, 2000 (EPS, Geneva, 2000), p. 1271.

[26] G. I. Budker, Proceedings of the CERN Symposium on High Energy Accelerators, CERN, Geneva, 1956, Vol. 1, p. 68 .

[27] A. V. Burov, V. I. Kudelainen, V. A. Lebedev, V. V. Parkhomchuk, A. A. Sery, and V.D. Shiltsev, Report No. INP 89-116, 1989; CERN Report No. CERN/PS 93-03 (AR), 1993.

[28] V. Shiltsev, A. Valishev, G. Kuznetsov, V. Kamerdzhiev, and A. Romanov, in Proceedings of the 23rd Particle Accelerator Conference, Vancouver, Canada, 2009 (IEEE, Piscataway, NJ, 2009), p. 3233.

[29] S. Nagaitsev et al., in Proceedings of the 18th Particle Accelerator Conference, New York, 1999 (IEEE, Piscataway, NJ, 1999), p. 521.

[30] S. Nagaitsev, A. Burov, A. C. Crawford, T. Kroc, J. MacLachlan, G. Saewert, C. W. Schmidt, A. Shemyakin, and A. Warner, Nucl. Instrum. Methods Phys. Res., Sect. A441, 241 (2000).

[31] P. Wesolowski, K. Balewski, R. Brinkmann, Y. Derbenev, and K. Floettmann, Nucl. Instrum. Methods Phys. Res., Sect. A 441, 281 (2000).

[32] G. I. Budker et al., Part. Accel. 7, 197 (1976).

[33] N. Dikansky, S. Nagaitsev, and V. Parkhomchuk, Fermilab Report No. FERMILAB-TM-1998-H, 1996.

[34] N. Dikansky et al., in Proceedings of the Particle Accelerator ConferenceVancouver, BC, Canada, 1997 (IEEE, Piscataway, NJ, 1997), p. 1795.

[35] A. Burov, V. Danilov, Ya. Derbenev, and P. Colestock, in Proceedings of the Workshop on Electron Cooling and Related Topics Incorporating the 5th Workshop on Medium Energy Electron Cooling, Uppsala, Sweden, 1999 (Elsevier Science, Amsterdam, 2000), p. 271.

[36] Y. Derbenev, in Proceedings of the Workshop on Electron Cooling and Related Topics Incorporating the 5th Workshop on Medium Energy Electron Cooling, Uppsala, Sweden, 1999 (Elsevier Science, Amsterdam, 2000), p. 223.

[37] D. Kehne, K. Low, M. Reiser, T. Shea, C. R. Chang, and Y. Chen, Nucl. Instrum. Methods Phys. Res., Sect. A 278, 194 (1989).

[38] S. Humphries, Jr and L. K. Len, J. Appl. Phys. 62, 1568 (1987).

[39] P. Loschialpo, W. Namkung, M. Reiser, and J. D. Lawson, J. Appl. Phys. 57, 10 (1985); http://dx.doi.org/10.1063/1 .335382 .

[40] W. Fischer et al., in Proceedings of the 24th Particle Accelerator Conference, PAC-2011, New York, 2011 (IEEE, New York, 2011), p. 2223.

[41] V. Shiltsev and D. Finley, Report No. FERMILAB-TM2008, 1997.

[42] V. Shiltsev, Report No. FERMILAB-TM-2031, 1997. 
[43] V. Shiltsev, V. Danilov, D. Finley, and A. Sery, Report No. FNAL-Pub-98/260, 1998.

[44] V Shiltsev et al., New J. Phys. 10, 043042 (2008).

[45] A. Burov, V. Danilov, and V. Shiltsev, Phys. Rev. E 59, 3605 (1999).

[46] L. Adamczyk et al., Phys. Rev. Lett. 112, 032302 (2014).

[47] P. Sorensen et al., RHIC Critical Point Search: Assessing STAR's Capabilities, http://arxiv.org/pdf/nucl-ex/ 0701028v1.pdf.

[48] Evidence of a QCD critical endpoint at RHIC, http:// marcofrasca.wordpress.com/2011/06/21/evidence-of-a-qcdcritical-endpoint-at-rhic/.

[49] C. Montag et al., in Proceedings of the 4th International Particle Accelerator Conference, IPAC-2013, Shanghai, China, 2013 (JACoW, Shanghai, China, 2013), p. 1523, http://accelconf.web.cern.ch/AccelConf/IPAC2013/ papers/tupfi076.pdf.

[50] G. Odyniec, Proc. Sci., CPOD2013 (2013) 043.

[51] C. Montag and A. Fedotov, Proc. Sci., CPOD (2013) 044.

[52] A. Fedotov and M. Blaskiewicz, Report No. C-A/AP/\#449, 2012.

[53] I. Hofmann, G. Franchetti, O. Boine-Frankenheim, J. Qiang, and R. D. Ryne, Phys. Rev. ST Accel. Beams 6, 024202 (2003).

[54] A. V. Fedotov and I. Hofmann, Phys. Rev. ST Accel. Beams 5, 024202 (2002).

[55] A. V. Fedotov, J. Holmes, and R. L. Gluckstern, Phys. Rev. ST Accel. Beams 4, 084202 (2001).

[56] V. Kapin and Yu. Alexahin, in Proceedings of Russian Particle Accelerator Conference 2010 (IHEP, Protvino, 2010), pp. 204-206.

[57] V. Kapin, FNAL Report No. Beamsdoc-3582-v2, 2011.

[58] M. A. Furman, in Proceedings of the 1987 Particle Accelerator Conference (IEEE, Washington, DC, 1987), p. 1034.

[59] Y. Alexain et al., Report No. Beams-doc-2609, http:// beamdocs.fnal.gov.

[60] M. Aiba et al., in Proceedings of PAC 2007, Albuquerque, New Mexico, USA (IEEE, Piscataway, NJ, 2007), p. 3390.

[61] A Burov and V. Lebedev, Phys. Rev. ST Accel. Beams, 12, 034201 (2009).

[62] K. Y. Ng, Physics of Intensity Dependent Beam Instabilities (World Scientific, Singapore, 2006).

[63] H. Pfeffer and G. Saewert, Report No. FERMILAB-PUB11-425-AD.
[64] A. Pikin (private communication).

[65] B. Dunham et al., Appl. Phys. Lett. 102, 034105 (2013); http://dx.doi.org/10.1063/1.4789395.

[66] S. Belomestnykh, Status of BNL SRF guns, ERL 2011 Workshop KEK, Tsukuba, Japan, 2011, http://accelconf .web.cern.ch/AccelConf/ERL2011/talks/wg1001_talk.pdf.

[67] A. V. Fedotov et al., in Proceedings of COOLO9 Workshop, Lanzhou, China, http://accelconf.web.cern.ch/ AccelConf/COOL2009/papers/mom2mcio01.pdf.

[68] A. V. Fedotov et al., in Proceedings of the 25th Particle Accelerator Conference, PAC-2013, Pasadena, CA, 2013 (IEEE, New York, 2013), p. 363, http://jacow.web.psi.ch/ conf/pac13/prepress/TUOAA1.PDF.

[69] K. Schindl, in Proceedings of the Joint U.S.-CERN-JapanRussia School on Particle Accelerators, Beam Measurements, Montreux, 1998, edited byS. Kurokawa, S. Y. Lee, E. Perevedentsev, and S. Turner (World Scientific, Singapore, 1999), pp. 127-151.

[70] R. L. Gluckstern, FERMILAB Note No. TM-1402, 1986, http://inspirehep.net/record/231017/files/fermilab-tm-1402 .PDF.

[71] L. J. Laslett, On Intensity Limitations Imposed by Transverse Space-Charge Effects in Circular Particle Accelerators, eConf C630610, 324 (1963), http:// inspirehep.net/record/48302? ln=en.

[72] K. Takayama, Lett. Nuovo Cimento 34, 190 (1982).

[73] L. D. Landau and E. M. Liftshitz, The Classical Theory of Fields (Pergamon Press, New York, 1994).

[74] M. Abramowitz and I. A. Stegun, Handbook of Mathematical Functions (National Bureau of Standards, Washington, DC, 1964).

[75] V. N. Litvinenko, Report No. C-A/AP/406, 2010, http:// www.cadops.bnl.gov/AP/ap_notes/ap_note_406.pdf.

[76] V. N. Litvinenko, Selected Notes from the USPAS Winter 2008 Accelerator Physics Course, 2010, pp. 1-116, http:// uspas.fnal.gov/materials/08UCSC/Accelerator_Physics1 .pdf.

[77] S. Kheifets, PETRA Note No. 119, 1976.

[78] V. Shiltsev et al., Phys. Rev. ST Accel. Beams 11, 103501 (2008).

[79] V. Shiltsev,V. Danilov, D. Finley, and A. Sery, Phys. Rev. ST Accel. Beams, 2, 071001 (1999).

[80] A. Burov, S. Nagaitsev, A. Shemyakin, and Ya. Derbenev, Phys. Rev. ST Accel. Beams 3, 094002 (2000). 